On the calculation of the D-optimal multisine excitation power spectrum for broadband impedance spectroscopy measurements

This article has been downloaded from IOPscience. Please scroll down to see the full text article.

2012 Meas. Sci. Technol. 23085702

(http://iopscience.iop.org/0957-0233/23/8/085702)

View the table of contents for this issue, or go to the journal homepage for more

Download details:

IP Address: 147.83.49.133

The article was downloaded on 03/09/2012 at 11:06

Please note that terms and conditions apply. 


\title{
On the calculation of the $D$-optimal multisine excitation power spectrum for broadband impedance spectroscopy measurements
}

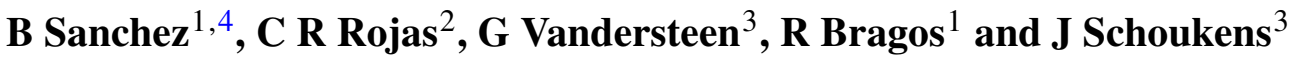 \\ ${ }^{1}$ Electronic and Biomedical Instrumentation Group, Department of Electrical Engineering, \\ Universitat Politecnica de Catalunya (UPC), 08034 Barcelona, Spain \\ ${ }^{2}$ Automatic Control Lab and ACCESS Linnaeus Center, School of Electrical Engineering, \\ KTH-Royal Institute of Technology, SE-100 44 Stockholm, Sweden \\ ${ }^{3}$ Department of Fundamental Electricity (ELEC), Vrije Universiteit Brussel (VUB), B-1050 Brussels, \\ Belgium \\ E-mail: benjamin.sanchez@upc.edu
}

Received 7 April 2012, in final form 22 May 2012

Published 4 July 2012

Online at stacks.iop.org/MST/23/085702

\begin{abstract}
The successful application of impedance spectroscopy in daily practice requires accurate measurements for modeling complex physiological or electrochemical phenomena in a single frequency or several frequencies at different (or simultaneous) time instants. Nowadays, two approaches are possible for frequency domain impedance spectroscopy measurements: (1) using the classical technique of frequency sweep and (2) using (non-)periodic broadband signals, i.e. multisine excitations. Both techniques share the common problem of how to design the experimental conditions, e.g. the excitation power spectrum, in order to achieve accuracy of maximum impedance model parameters from the impedance data modeling process. The original contribution of this paper is the calculation and design of the $D$-optimal multisine excitation power spectrum for measuring impedance systems modeled as $2 \mathrm{R}-1 \mathrm{C}$ equivalent electrical circuits. The extension of the results presented for more complex impedance models is also discussed. The influence of the multisine power spectrum on the accuracy of the impedance model parameters is analyzed based on the Fisher information matrix. Furthermore, the optimal measuring frequency range is given based on the properties of the covariance matrix. Finally, simulations and experimental results are provided to validate the theoretical aspects presented.
\end{abstract}

Keywords: electrical impedance spectroscopy (EIS), multisine excitation, input, signals, experiment design, electrical bio-impedance (EBI)

\section{Introduction}

Nowadays, techniques based on electrical/electrochemical impedance spectroscopy (EIS) provide valuable information in a wide range of applications, i.e. from in/ex vivo tissue and organ characterization [1-4] to the recording of information about biological events occurring at the biosensor electrode

\footnotetext{
${ }^{4}$ Author to whom any correspondence should be addressed.
}

surfaces, inducing capacitance and resistance changes [5, 6]. The characterization of biological materials through EIS, known as electrical bio-impedance (EBI), provides relevant physiological information about the properties of biological materials as a function of the excitation frequency, which is related, at a macroscopic level, to the physiological states of tissues and, at a molecular level, to the cell size and shape, cell membrane state, cell concentration and intra- and extracellular compartments among others. In the electrochemical field, 
when a biological receptor binds to its counterpart attached to the surface of a biosensor, there is a change in the impedance of the system that enables direct measurement of an electrical signal [7-9]. To measure the impedance frequency response, most frequency domain approaches are based on commercial impedance analyzers implementing single impedance spectrum frequency measurements or on frequency sweep (using linear or logarithmic steps) [10].

In order to reduce the measurement time, researchers have proposed methods without frequency scanning. An overview of signal design and applications for system identification can be found in [11]. First measurements of full impedance spectra were not made until approximately the 1970s, with the publication reported in [12]. Their approach was based on mixing ac waves of many different frequencies superimposed on a desired dc bias potential to an electrochemical system. The use of the random phase multisine signal was introduced by Creason et al and Schwall et al in [13, 14], respectively. Around the same time, a maximum length binary sequence generator based on a shift register for characterizing RC circuits was presented in [15]. More recent applications can be found where multisine excitations [16] have been used in both electrochemical and EBI spectroscopy applications in [17-22] and [23-25], respectively. The steady-state [26] or non-stationary [27] impedance response is measured and its frequency response is usually estimated using the discrete Fourier transform.

The successful application of broadband impedance spectroscopy measurements in everyday practice relies on fast and accurate impedance measurements for the extraction of relevant parameters from the modeling process. However, this accuracy is compromised due to the short measuring time when measuring with broadband signals [28]. As shown in this paper, this loss can be mitigated by optimizing the excitation in the frequency domain. The advantages offered by using optimal input signals are as follows.

(i) Making the optimal analysis helps the engineer to obtain valuable information in what frequency bands the system is most open to collect information.

(ii) Optimal signals contribute to maximize the amount of information from the system under test.

(iii) It would help to improve the performance of commercial impedance measurement equipment.

A feature of the optimal experimental framework is that a priori information of the model is needed to be able to optimize the excitation. Fortunately, this knowledge can be easily obtained if a two-step process impedance measurement is performed. Firstly, measurements are carried out to obtain a general overview of the system and a preliminary model for describing the acquired data. This model is then used for improving the excitation time-frequency features in order to increase the impedance measurement accuracy for the second step of measurements. Nevertheless, optimal input signals can also create severe risks. Special care must be taken in those cases where the model considered for the excitation optimization framework does not exactly describe the reality. If so, exciting with the optimal excitation can be counterproductive since there exists the risk of losing information. To overcome these situations, a more robust design is required [29-33].

The aim of this work is to design and analyze the $D$-optimal multisine excitation for broadband impedance spectroscopy measurements. The influence of the excitation power spectrum on the properties of the Fisher information matrix is investigated for $2 \mathrm{R}-1 \mathrm{C}$ impedance models. To succeed in this, the basics for the $D$-optimal input design are presented in section 2 and illustrated through an example in section 2.1. Once the reader is familiar with the theoretical concepts of the experiment design field, the $D$-optimal framework is applied to the study of a $2 \mathrm{R}-1 \mathrm{C}$ impedance model in section 3 . The dependence of the $D$-criterion on the multisine frequencies and power amplitude spectrum is evaluated in sections 4.1 and 4.2 respectively. A variance analysis on the estimated impedance model parameters as a function of the multisine excitation frequencies is presented in section 4.3. Next, section 4.4 deals with the estimation of the impedance model parameters considering an output error framework when exciting with the $D$-optimal and a non-optimal random phase multisine. Section 5 shows the estimated impedance model parameters from data measured using a custom broadband impedance analyzer. Finally, the results obtained are discussed in section 6 and summarized in the form of conclusions in section 7 .

\section{Theoretical background revised}

Consider an (impedance) system modeled by $Z(s)$, where $s$ is the complex argument $(s=\mathrm{j} \omega)$ and the Fisher information matrix $M(\omega)$ with respect to the model vector parameters $\theta$ is defined under the standard Gaussian noise condition as [34-36, 31]

$$
M(\omega) \triangleq \mathfrak{R e}\left\{\frac{\partial Z(\mathrm{j} \omega)}{\partial \theta} \cdot\left(\frac{\partial Z(\mathrm{j} \omega)}{\partial \theta}\right)^{H}\right\},
$$

where the superscript $H$ denotes the conjugate transpose operator and $\omega$ the angular frequency. It can be shown that, according to the Cramér-Rao inequality, the parameter covariance matrix of every unbiased estimator is lower bounded by the inverse of the Fisher information matrix, i.e.

$$
\operatorname{cov} \hat{\theta}(\omega) \geqslant M(\omega)^{-1}
$$

and that this bound is asymptotically achievable under mild conditions [31].

The reader should note that $M(\omega)$ is a matrix by definition. The Fisher information matrix, defined by (1), directly depends on the sensitivity of $Z(\mathrm{j} \omega ; \theta)$ with respect to the vector $\theta$. This makes sense, since the estimation procedure can be seen as an inverse problem, where the direct problem is as follows: given $\theta$, generate data from $Z(\mathrm{j} \omega ; \theta)$. Evidently, the larger $\partial Z(\mathrm{j} \omega, \theta) / \partial \theta$ is (according to some scalar measure of $\partial Z(\mathrm{j} \omega, \theta) / \partial \theta)$, the more different the data will look for different values of $\theta$. This means that the larger $\partial Z(\mathrm{j} \omega, \theta) / \partial \theta$ is, the easier it becomes to estimate the correct $\theta$ which generated the data, and this is reflected in a smaller variance of an (asymptotically efficient) estimate of $\theta$. 

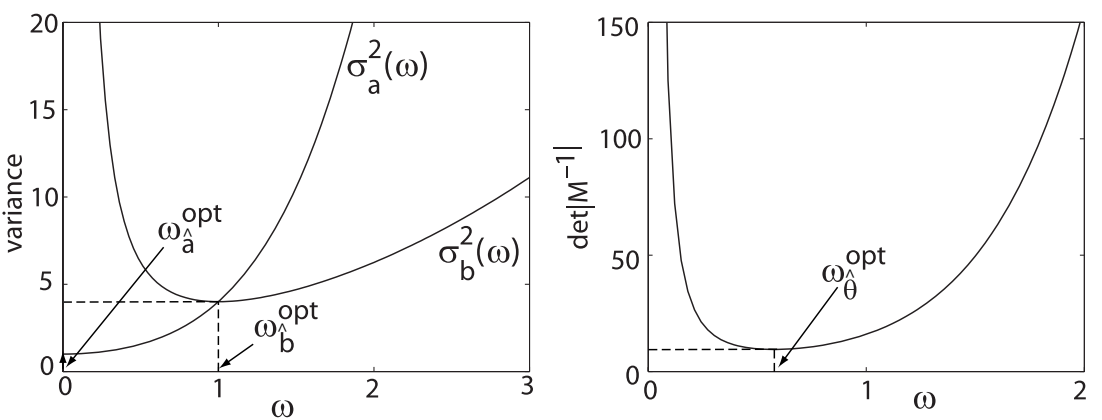

Figure 1. The (normalized by $N$ ) variance of the parameters as a function of the input excitation angular frequency $\omega$, where the true values are $a=b=1$ (left). Determinant of the Cramér-Rao lower bound as a function of the angular frequency of excitation $\omega$ (right).

\subsection{Example: first-order linear dynamic impedance system}

This section illustrates the influence of the input sinewave excitation frequency $\omega$ on the variance of the impedance model parameters. Consider the modeling of a first-order linear dynamic impedance system given by

$$
Z(s)=\frac{1}{a+b s},
$$

where $\theta=[a b]^{T}$ are the parameters to be estimated. The system is excited with amplitude 1 at angular frequency $\omega$. The parameters $[a b]$ have to be estimated from noisy observations of the input-output Fourier coefficients. The noise is assumed to be independent and normally distributed, with zero mean and unit variance. Considering the impedance model given by (3) for the calculation of the Fisher information matrix defined by (1), namely

$$
\frac{\partial Z(\mathrm{j} \omega)}{\partial \theta}=\left[\begin{array}{c}
\frac{-1}{(a+\mathrm{j} \omega b)^{2}} \\
\frac{-\mathrm{j} \omega}{(a+\mathrm{j} \omega b)^{2}}
\end{array}\right],
$$

it can be found that the first and second diagonal Fisher information matrix elements $M_{11}(\omega)$ and $M_{22}(\omega)$ are

$$
\begin{aligned}
& M_{11}(\omega)=\mathfrak{R e}\left\{\frac{\partial Z(\mathrm{j} \omega)}{\partial a}\left(\frac{\partial Z(\mathrm{j} \omega)}{\partial a}\right)^{H}\right\}=\frac{1}{\left(a^{2}+\omega^{2} b^{2}\right)^{2}} \\
& M_{22}(\omega)=\mathfrak{R e}\left\{\frac{\partial Z(\mathrm{j} \omega)}{\partial b}\left(\frac{\partial Z(\mathrm{j} \omega)}{\partial b}\right)^{H}\right\}=\frac{\omega^{2}}{\left(a^{2}+\omega^{2} b^{2}\right)^{2}},
\end{aligned}
$$

while the terms $M_{12}(\omega)$ and $M_{21}(\omega)$ are zero. Therefore, the matrix notation for the Fisher information matrix for $N$ samples is

$$
M(\omega)=\frac{N}{\left(a^{2}+b^{2} \omega^{2}\right)^{2}}\left[\begin{array}{cc}
1 & 0 \\
0 & \omega^{2}
\end{array}\right] .
$$

The Fisher information matrix $M(\omega)$ for the first-order linear dynamic system reflects the fact that, asymptotically in the number of samples $N$, asymptotically efficient estimates of $a$ and $b$ will be mutually independent (this follows from the diagonal structure of $M(\omega)$ ). The estimation of $b$ becomes increasing better/easier than that of $a$ for larger $\omega$. On the other hand, the common denominator increases like $\omega^{4}$, which dominates for large $\omega$. This means that while the best estimates of $a$ can be obtained by putting a constant signal $(\omega=0)$, there is an optimal non-zero frequency $\omega^{*}\left(\omega^{*}=a / b\right.$, obtained by differentiating $M_{22}$ with respect to $\omega^{2}$ and setting it equal to zero), where $M_{22}$ stands for the second diagonal element of the Fisher information matrix. The reader should note, however, that a constant signal would not allow a consistent estimation of $b$ due to the lack of sufficient persistence of excitation [37], while a sinusoid of frequency $\omega^{*}$, which has an order of persistence of excitation equal to 2, would enable the consistent estimation of both $a$ and $b$. In fact, the inverse of the Fisher information matrix is

$$
\operatorname{cov} \hat{\theta}(\omega)=M(\omega)^{-1}=\frac{\left(a^{2}+b^{2} \omega^{2}\right)^{2}}{N}\left[\begin{array}{cc}
1 & 0 \\
0 & 1 / \omega^{2}
\end{array}\right] .
$$

The choice of the experimental angular frequency will depend on which parameter is considered to be the most important: as can be observed from figure 1 (left), for $a$ alone $\omega_{\widehat{a}}^{\mathrm{opt}}=0$ would be the best, while for $b$ alone $\omega_{\widehat{b}}^{\mathrm{opt}}=1 \mathrm{rad} \mathrm{s}^{-1}$ would be the best.

It may also happen that we are interested in estimating with maximum accuracy all model parameters, this is, $a$ and $b$. In such a case, the determinant of the covariance matrix (see (7)) can be used to evaluate the joint accuracy of both impedance model parameters $\theta=[a b]^{T}$; this is the socalled $D$-criterion. Due to the diagonal nature of $M$ in the first-order example, the $D$-criterion corresponds to the sum of the logarithms of the variances of $\hat{a}$ and $\hat{b}$. This gives figure 1 (right) as a combination of the plots $\sigma_{a}^{2}$ and $\sigma_{b}^{2}$ from figure 1 (left). For this case, the angular frequency where to optimally estimate both parameters is $\omega_{\hat{\theta}}^{\text {opt }}=0.57 \mathrm{rad} \mathrm{s}^{-1}$. With this example, the reader must realize how important it is to choose the excitation frequencies: depending on the excitation frequencies, the impedance model parameters estimated will have greater or lower variance, directly affecting the quality of extraction of relevant information from the model for its subsequent interpretation. Section 3 focuses on studying the same concepts but for an impedance 2R-1C model.

\section{Case study: 2R-1C impedance model}

The previous section showed how important it is to carefully choose the excitation frequency in order to obtain accurate impedance model estimates. Let us now consider an impedance model $Z(s)$ described by the following transfer function:

$$
Z(s)=\frac{a s+b}{c s+1}
$$

modeled as a zero-pole continuous time system. This assumption enables the system to be modeled as an electrical 

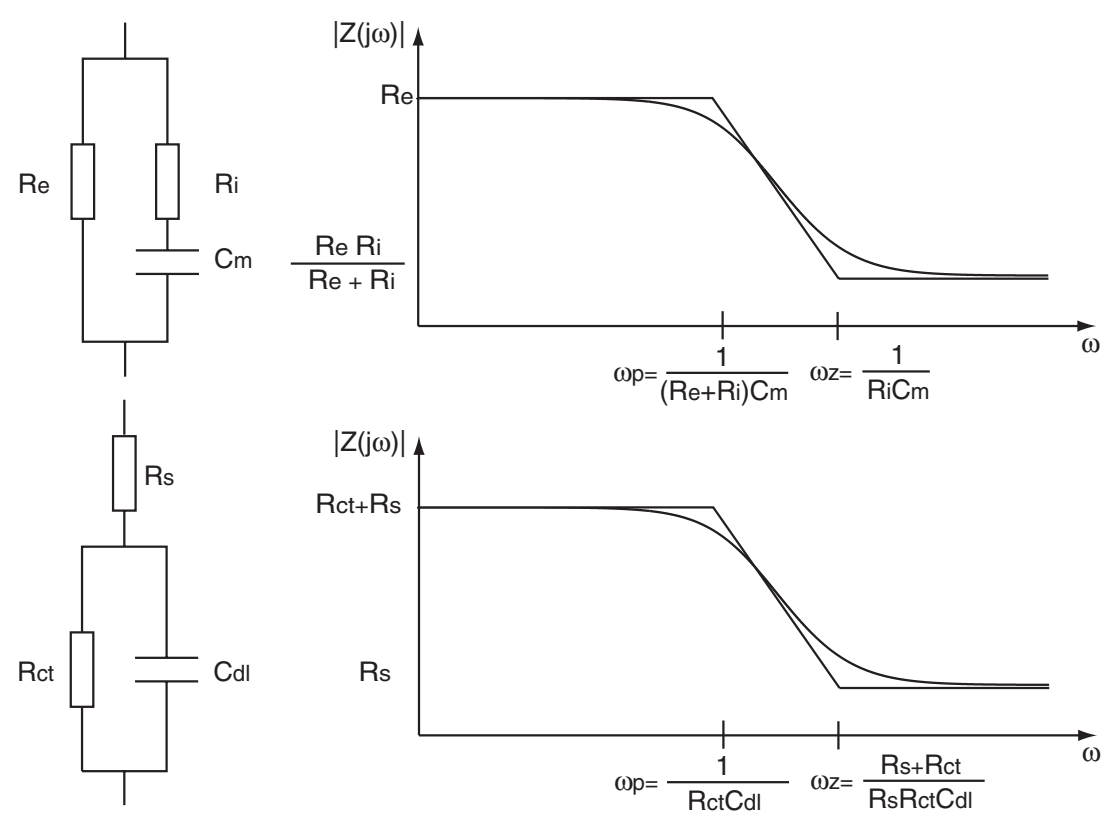

Figure 2. Equivalent electrical (left) bio-impedance (top) and electrochemical (bottom) circuit representation and impedance magnitude Bode plot (right). The Bode plot shows the dc and infinite values for the magnitude and the position of the cutoff frequencies, $\omega_{z}$ and $\omega_{p}$, as a function of each of the circuit parameters. See the text for details.

circuit with three components: two resistors and one capacitor, the so-called $2 \mathrm{R}-1 \mathrm{C}$, as shown in figure 2.

The meaning of each of the impedance model parameters $\theta=[a b c]^{T}$ depends on the application. In EBI applications, (8) is commonly written as a function of the equivalent electrical circuit parameters that model suspended living cells; these are $R_{e}$, related to the impedance of the extracellular liquid, $R_{i}$, the resistance related to the intracellular liquid, and $C_{m}$, a capacitor that models the capacitance frequency behavior of the cell membrane [38]. This bio-impedance model corresponds to a series $R_{i} C_{m}$ electrical circuit in parallel with the resistor $R_{e}$ (see figure 2, top left). Since this is a simple impedance transfer function, there is just one possible solution relating the position of the impedance transfer function pole and zero (see (8)) to the three equivalent electrical circuit parameters $R_{e}, R_{i}$ and $C_{m}$ as follows:

$$
\begin{aligned}
& a=R_{e} R_{i} C_{m} \\
& b=R_{e} \\
& c=\left(R_{e}+R_{i}\right) C_{m}
\end{aligned}
$$

and its frequency response is shown in figure 2 (top right).

On the other hand, a series circuit bridge can be found from the same model given by (8), which is frequently used to model skin tissue bio-impedances as well as to interpret electrochemical impedance spectra. The Randles circuit [39] shown in figure 2 (bottom left) is formed by an equivalent electrical circuit consisting of an active electrolyte resistance $R_{S}$ in series with the parallel combination of the double-layer capacitance $C_{d l}$ and an active charge transfer resistance $R_{c t}$. In this case, the relation to the impedance model parameters defined in (8) is identified by

$$
\begin{aligned}
& a=R_{s} R_{c t} C_{d l} \\
& b=R_{s}+R_{c t} \\
& c=R_{c t} C_{d l} .
\end{aligned}
$$

Because both electrical circuits are equivalent (they are defined by the same unique impedance transfer function $Z(s)$ ), any conclusion derived from the $D$-optimization framework applied to the impedance model given by (8) will be valid for electrical/electrochemical (bio-)impedance multisine spectroscopy measurements.

\subsection{D-optimal design}

Let (8) be the impedance frequency response to be estimated, where $\theta=\left[\begin{array}{lll}a & b & c\end{array}\right]^{T}$ is the impedance model parameter vector. Then,

$$
\frac{\partial Z(\mathrm{j} \omega)}{\partial \theta}=\left[\begin{array}{c}
\frac{\mathrm{j} \omega}{\mathrm{j} \omega c+1} \\
\frac{1}{\mathrm{j} \omega c+1} \\
\frac{-\mathrm{j} \omega(\mathrm{j} \omega a+b)}{(\mathrm{j} \omega c+1)^{2}}
\end{array}\right]
$$

which implies that

$$
\frac{\partial Z(\mathrm{j} \omega)}{\partial \theta}=\frac{1}{(\mathrm{j} \omega c+1)^{2}} \underbrace{\left[\begin{array}{ccc}
0 & 1 & c \\
1 & c & 0 \\
0 & -b & -a
\end{array}\right]}_{\triangleq T}\left[\begin{array}{c}
1 \\
\mathrm{j} \omega \\
(\mathrm{j} \omega)^{2}
\end{array}\right] .
$$

Therefore, the Fisher information matrix can be calculated using (1) and rewritten in terms of the $T$ matrix given in (12) as follows:

$$
M(\omega)=\frac{1}{\left(1+c^{2} \omega^{2}\right)^{2}} T\left[\begin{array}{ccc}
1 & 0 & -\omega^{2} \\
0 & \omega^{2} & 0 \\
-\omega^{2} & 0 & \omega^{4}
\end{array}\right] T^{T} .
$$

Finally [40],

$$
M(\omega)=\left[\begin{array}{ccc}
\frac{\omega^{2}}{1+\omega^{2} c^{2}} & 0 & -\frac{\omega^{2}\left(b+a c \omega^{2}\right)}{\left(1+c^{2} \omega^{2}\right)^{2}} \\
0 & \frac{1}{1+\omega^{2} c^{2}} & \frac{(a-b c) \omega^{2}}{\left(1+c^{2} \omega^{2}\right)^{2}} \\
-\frac{\omega^{2}\left(b+a c \omega^{2}\right)}{\left(1+c^{2} \omega^{2}\right)^{2}} & \frac{(a-b c) \omega^{2}}{\left(1+c^{2} \omega^{2}\right)^{2}} & \frac{\omega^{2}\left(b^{2}+a^{2} \omega^{2}\right)}{\left(1+c^{2} \omega^{2}\right)^{2}}
\end{array}\right]
$$


which can be evaluated at $\omega=0$ :

$$
M(0)=\left[\begin{array}{lll}
0 & 0 & 0 \\
0 & 1 & 0 \\
0 & 0 & 0
\end{array}\right]
$$

and for $\omega \gg 1$ :

$$
\begin{aligned}
\left.M(\omega)\right|_{\omega \gg 1} & \approx \frac{1}{c^{4} \omega^{4}}\left[\begin{array}{ccc}
c^{2} \omega^{4} & 0 & -a c \omega^{4} \\
0 & c^{2} \omega^{2} & \omega^{2}(a-b c) \\
-a c \omega^{4} & \omega^{2}(a-b c) & a^{2} \omega^{4}
\end{array}\right] \\
& \approx \frac{1}{c^{4}}\left[\begin{array}{ccc}
c^{2} & 0 & -a c \\
0 & 0 & 0 \\
-a c & 0 & a^{2}
\end{array}\right]
\end{aligned}
$$

From (14), it can be observed that the Fisher information matrix for the $2 \mathrm{R}-1 \mathrm{C}$ impedance model is more complicated to analyze than for the first-order example shown in section 2.1, since it is not diagonal. This fact implies that the estimates of $a$, $b$ and $c$ are correlated. However, the fact that $M_{12}=M_{21}=0$, where $M_{i j}$ stands for the Fisher information matrix elements situated at row $i$ column $j$, can be interpreted as stating that $\hat{a}$ and $\hat{b}$ are (asymptotically) conditionally independent given $c$, i.e. conditioned to the event that $\hat{c}$ is fixed to a given value, $\hat{a}$ and $\hat{b}$ are independent. This is similar to the first-order example case shown in section 2.1, where $Z$ depends on a linear combination of $a$ and $b$.

This shows that excitations of low frequency can give rise to good estimates of $b$ (but render $a$ and $c$ unidentifiable), while excitations of high frequency contribute to the estimation of $a / c$ (but render $b$ unidentifiable). A direct interpretation of these results can be derived from a dc/ac analysis of the bio-impedance RC-circuit model shown in figure 2 (top): the capacitor $C_{m}$ behaves as an open circuit at low frequencies; hence, $Z$ only depends on $R_{e}$, while at high frequencies, $C_{m}$ behaves like a short circuit, which means that $Z$ depends mainly on the parallel combination $R_{e} R_{i} /\left(R_{e}+R_{i}\right)=a / c$. The interpretation is similar for the case of the impedance Randles circuit model [39] shown in figure 2 (bottom): at low frequencies, $Z$ depends on $R_{c t}+R_{s}$, while at high frequencies $Z$ depends mainly on $R_{s}$. Both explanations can be justified from the impedance transfer function given by (8) by evaluating the angular frequency $\omega$ at very high and low frequencies. It follows that $\left.Z(\mathrm{j} \omega)\right|_{\omega \rightarrow 0}=b$ and $\left.Z(\mathrm{j} \omega)\right|_{\omega \rightarrow \infty}=a / c$. As mentioned before, the low and high frequencies give information on $b$ and $a / c$, respectively. To isolate the values of $a$ and $c$, intermediate excitation frequencies are needed.

At this point, the reader should not forget what is the goal, which is to design the $D$-optimal input excitation power spectrum based on the Fisher information matrix. For this, it is necessary to introduce the concept of full Fisher information matrix which is defined as

$$
\bar{M}=2 \int_{0}^{\infty} M(\omega) \Phi_{u}(\omega) \mathrm{d} \omega,
$$

where $\Phi_{u}$ is the input excitation power spectrum, either current or voltage depending on the measurement setup being used. The factor 2 in (17) comes from the fact that we are integrating only in the positive range of $\omega$. Since $M(\omega)$ and $\Phi_{u}(\omega)$ are even functions, their contribution to $\omega$ negative is the same as for $\omega$ positive, so for simplicity, we have restricted the integration interval (which in principle should cover all $\mathbb{R}$ ) to the positive $\omega$-axis, and added a factor 2 .

By definition, the full Fisher information matrix is a matrix. Because of that, a measure of the efficiency for the purpose of experiment design must be expressed as a scalar function of $\bar{M}$. There are several criteria available for measuring the size of the full Fisher information matrix, e.g. $A$-optimality criterion [41] $\left((\operatorname{tr} \bar{M})^{-1}\right)$ and $D$-optimality criterion [42], which is the approach presented in this paper. Using (13), the full Fisher information matrix can be rewritten in terms of the matrix $T$ as follows:

$\bar{M}=2 T\left\{\int_{0}^{\infty}\left[\begin{array}{ccc}1 & 0 & -\omega^{2} \\ 0 & \omega^{2} & 0 \\ -\omega^{2} & 0 & \omega^{4}\end{array}\right] \frac{\Phi_{u}(\omega)}{\left(1+c^{2} \omega^{2}\right)^{2}} \mathrm{~d} \omega\right\} T^{T}$.

For the sake of notational simplicity, the input excitation power spectrum can be rewritten in terms of

$$
\bar{\Phi}_{u}(\omega)=\frac{\Phi_{u}(\omega)}{\left(1+c^{2} \omega^{2}\right)^{2}} .
$$

If we are interested in the $D$-optimal excitation input power spectrum, then this can be formulated as the optimal input power spectrum $\Phi_{u}(\omega)$ that maximizes the determinant of the full Fisher information matrix, namely:

$$
\begin{aligned}
& \max _{\Phi_{u}} \operatorname{det}(\bar{M}) \\
& \quad=\max _{\bar{\Phi}_{u}} \operatorname{det}\left\{\int_{0}^{\infty}\left[\begin{array}{ccc}
1 & 0 & -\omega^{2} \\
0 & \omega^{2} & 0 \\
-\omega^{2} & 0 & \omega^{4}
\end{array}\right] \bar{\Phi}_{u}(\omega) \mathrm{d} \omega\right\}
\end{aligned}
$$

s.t. $\Phi_{u}(\omega) \geqslant 0, \forall \omega \geqslant 0$ with a constraint that restricts the amount of injected power to the system, namely

$$
\int_{0}^{\infty} \Phi_{u}(\omega) \mathrm{d} \omega=\int_{0}^{\infty}\left(1+2 c^{2} \omega^{2}+c^{4} \omega^{4}\right) \bar{\Phi}_{u}(\omega) \mathrm{d} \omega \leqslant K .
$$

In biomedical applications, this restriction on the amount of injected power makes sense since it is directly related to the safety regulations for bio-impedance measurements in humans. Since the estimation of the impedance model parameters $\theta$ is improved by scaling the input to increase its power, it follows that the $D$-optimal input should use all available power, i.e. the power constraint should hold as an equality. Also, $T$ and $T^{T}$ matrices from (18) as well as the factor 2 only contribute to $\operatorname{det}(\bar{M})$ as constant factors, which is the reason why they can be omitted in the analysis. Here the reader should realize that the optimal spectrum depends only on $c$ (but not on $a$ nor $b$ ). The reader should note also that (21) depend on three quantities related to $\bar{\Phi}_{u}$ :

$$
\begin{array}{r}
r_{0}=\int_{0}^{\infty} \bar{\Phi}_{u}(\omega) \mathrm{d} \omega \\
r_{2}=\int_{0}^{\infty} \omega^{2} \bar{\Phi}_{u}(\omega) \mathrm{d} \omega \\
r_{4}=\int_{0}^{\infty} \omega^{4} \bar{\Phi}_{u}(\omega) \mathrm{d} \omega .
\end{array}
$$


On the other hand, the non-negativity condition of $\bar{\Phi}_{u}$ defined in (20) holds if

$$
\max _{r_{0}, r_{2}, r_{4}} \operatorname{det}\left[\begin{array}{ccc}
r_{0} & 0 & -r_{2} \\
0 & r_{2} & 0 \\
-r_{2} & 0 & r_{4}
\end{array}\right] \text { s.t. }\left[\begin{array}{ccc}
r_{0} & 0 & r_{2} \\
0 & r_{2} & 0 \\
r_{2} & 0 & r_{4}
\end{array}\right] \geqslant 0
$$

with $r_{0}+2 c^{2} r_{2}+c^{4} r_{4}=K$. This condition comes from the theory of the truncated Hamburger moment problem [43]. If the cost function given by (23) is written as

$$
J=-\log \operatorname{det}\left[\begin{array}{ccc}
r_{0} & 0 & -r_{2} \\
0 & r_{2} & 0 \\
-r_{2} & 0 & r_{4}
\end{array}\right]
$$

(to be minimized instead of maximized), then (23) becomes a standard convex optimization problem. To solve (24), we can use CVX, a package for specifying and solving convex programs $[44,45]$. For example, the CVX solution to (24) for $c=0.1$ and $K=1$ is

$$
\begin{aligned}
& r_{0}^{\mathrm{opt}}=0.3750 \\
& r_{2}^{\mathrm{opt}}=12.50 \\
& r_{4}^{\mathrm{opt}}=3.750 \times 10^{3} .
\end{aligned}
$$

\subsection{D-optimal multisine excitation power spectrum}

At this point, we have already numerically calculated the optimal values that minimize (24). Thus, the next point to address is the remaining problem for the power spectrum optimization, that is, given the optimal numerical values $r_{0}^{\text {opt }}$, $r_{2}^{\text {opt }}$ and $r_{4}^{\text {opt }}$ shown in (25), how to design the optimal multisine excitation power spectrum $\bar{\Phi}_{u}$. The first thing to consider is that, since we want to estimate three parameters, the input signal should be such that the Fisher information matrix is non-singular, which means that the excitation signal should be persistently exciting of order 3 . In other words, the reader must keep in mind that one single excitation frequency enables us to estimate two parameters. Then, the minimum number of (non-negative) frequencies required for estimating (8) is two, since three parameters need to be estimated. Then, let $\bar{\Phi}_{u}^{\mathrm{opt}}$ be the optimal excitation power spectrum defined as a multisine signal composed of two exciting frequencies $\omega_{1}$ and $\omega_{2}$ :

$$
\bar{\Phi}_{u}^{\mathrm{opt}}=\alpha \delta\left(\omega-\omega_{1}\right)+\beta \delta\left(\omega-\omega_{2}\right)
$$

with $\alpha, \beta, \omega_{1}, \omega_{2} \geqslant 0$, i.e. assume that the optimal spectrum has two spectral lines (in the non-negative axis) and $\alpha$ and $\beta$ are the amplitudes at the frequencies $\omega_{1}$ and $\omega_{2}$, respectively. Then, combining (26) with (22), we have

$$
\begin{aligned}
& r_{0}^{\mathrm{opt}}=\int_{0}^{\infty} \bar{\Phi}_{u}^{\mathrm{opt}}(\omega) \mathrm{d} \omega=\alpha+\beta \\
& r_{2}^{\mathrm{opt}}=\int_{0}^{\infty} \omega^{2} \bar{\Phi}_{u}^{\mathrm{opt}}(\omega) \mathrm{d} \omega=\alpha \omega_{1}^{2}+\beta \omega_{2}^{2} \\
& r_{4}^{\mathrm{opt}}=\int_{0}^{\infty} \omega^{4} \bar{\Phi}_{u}^{\mathrm{opt}}(\omega) \mathrm{d} \omega=\alpha \omega_{1}^{4}+\beta \omega_{2}^{4} .
\end{aligned}
$$

The reader should note that in (27), there are only three equations defined by four quantities. This means that we have one degree of freedom available, for example, to select $\omega_{1}$. For instance, if we fix $\omega_{1}=0$, then

$$
\begin{gathered}
r_{0}^{\mathrm{opt}}=\alpha+\beta \\
r_{2}^{\mathrm{opt}}=\beta \omega_{2}^{2} \\
r_{4}^{\mathrm{opt}}=\beta \omega_{2}^{4},
\end{gathered}
$$

namely

$$
\begin{aligned}
\omega_{2} & =\sqrt{\frac{r_{4}^{\mathrm{opt}}}{r_{2}^{\mathrm{opt}}}} \\
\beta & =r_{2}^{\mathrm{opt}}\left(\sqrt{\frac{r_{2}^{\mathrm{opt}}}{r_{4}^{\mathrm{opt}}}}\right)^{2}=\frac{\left(r_{2}^{\mathrm{opt}}\right)^{2}}{r_{4}^{\mathrm{opt}}} \\
\alpha & =r_{0}^{\mathrm{opt}}-\frac{\left(r_{2}^{\mathrm{opt}}\right)^{2}}{r_{4}^{\mathrm{opt}}} .
\end{aligned}
$$

Note that this solution satisfies $k, \omega_{1}, \omega_{2} \geqslant 0$ since the first constraint in (24) implies that $r_{0} r_{4}-r_{2}^{2} \geqslant 0$, which is equivalent to $\alpha \geqslant 0$ (for the suggested solution given by (29)).

Nevertheless, other solutions are also possible, like e.g. choosing positive values $\omega_{1}>0$ and setting the amplitudes of the two spectral lines $\Phi_{u}$ to have equal magnitude, say, $A$. This is a standard approach in many EBI as well as EIS applications, where the input excitation power spectrum amplitude is usually designed to be flat along all the excitation excitation frequencies. Moreover, by setting $\omega_{1}>0$ it is possible to avoid exciting the system with very low frequency components (or dc). A practical example where it is preferable to avoid injecting low frequency signals can be found in body impedance spectroscopy applications. Contrary to the first solution where $\omega_{1}^{\text {opt }}=0$, choosing $\omega_{1}>0$ prevents tissue stimulation and minimizes the skin-electrode impedance impact on measurement. Furthermore, this second solution simplifies the optimization of the multisine signal in the time domain by the minimization of the crest factor [46, 40]. This second assumption, $\omega_{1}^{\text {opt }}>0$, is discussed in section 6 and translated into the following equation:

$$
\begin{aligned}
& A=\alpha\left(c^{2} \omega_{1}^{2}+1\right)^{2} \\
& A=\beta\left(c^{2} \omega_{2}^{2}+1\right)^{2} .
\end{aligned}
$$

By combining (26) with (19), the $D$-optimal spectrum must satisfy

$$
\begin{aligned}
& \Phi_{u}^{\mathrm{opt}}(\omega)=\left(c^{2} \omega^{2}+1\right)^{2} \tilde{\Phi}_{u}(\omega) \\
& \Phi_{u}^{\mathrm{opt}}(\omega)=\alpha\left(1+c_{2} \omega_{1}^{2}\right)+\beta\left(1+c_{2} \omega_{2}^{2}\right) .
\end{aligned}
$$

The reader should note that the set of equations to solve, (27) and (30), now constitutes a system of five equations in four unknowns, i.e. it is overdetermined. The reason is that the total input power $K$ has been fixed beforehand in our formulation. This can be solved by noting that the decision variables $r_{0}^{\text {opt }}$, $r_{2}^{\text {opt }}$ and $r_{4}^{\text {opt }}$ depend linearly on $K$, namely

$$
\begin{aligned}
& r_{0}^{\mathrm{opt}}=K \tilde{r}_{0}^{\mathrm{opt}} \\
& r_{2}^{\mathrm{opt}}=K \tilde{r}_{2}^{\mathrm{opt}} \\
& r_{4}^{\mathrm{opt}}=K \tilde{r}_{4}^{\mathrm{opt}},
\end{aligned}
$$


where $\tilde{r}_{0}^{\text {opt }}, \tilde{r}_{2}^{\text {opt }}$ and $\tilde{r}_{4}^{\text {opt }}$ are the solutions of (23) for $K=1$. The quantity $K$ can now be considered as an additional variable, to be also determined. Therefore, the equations defining $\alpha, \beta$, $\omega_{1}, \omega_{2}, K$ are

$$
\begin{gathered}
K \tilde{r}_{0}^{\mathrm{opt}}=\alpha+\beta \\
K \tilde{r}_{2}^{\mathrm{opt}}=\alpha \omega_{1}^{2}+\beta \omega_{2}^{2} \\
K \tilde{r}_{4}^{\mathrm{opt}}=\alpha \omega_{1}^{4}+\beta \omega_{2}^{4} \\
A=\alpha c^{4} \omega_{1}^{4}+2 \alpha c^{2} \omega_{1}^{2}+\alpha \\
A=\beta c^{4} \omega_{2}^{4}+2 \beta c^{2} \omega_{2}^{2}+\beta .
\end{gathered}
$$

The system of equations can be solved by first summing $(33 d)$ and (33e) and substituting (33a)-(33c), which gives

$$
\begin{aligned}
2 A & =\alpha c^{4} \omega_{1}^{4}+\beta c^{4} \omega_{2}^{4}+2 \alpha c^{2} \omega_{1}^{2}+2 \beta c^{2} \omega_{2}^{2}+\alpha+\beta \\
& =c^{4} K \tilde{r}_{4}^{\mathrm{opt}}+2 c^{2} K \tilde{r}_{2}^{\mathrm{opt}}+K \tilde{r}_{0}^{\mathrm{opt}} \\
& =K\left[c^{4} \tilde{r}_{4}^{\mathrm{opt}}+2 c^{2} \tilde{r}_{2}^{\mathrm{opt}}+\tilde{r}_{0}^{\mathrm{opt}}\right] \\
& =K .
\end{aligned}
$$

The value of $K$ defined in (34) can be plugged into equations $(33 a)-(33 d)$. The solution can be found using numeric or analytical software, namely:

$$
\begin{gathered}
2 A \tilde{r}_{0}^{\mathrm{opt}}=\alpha+\beta \\
2 A \tilde{r}_{2}^{\mathrm{opt}}=\alpha \omega_{1}^{2}+\beta \omega_{2}^{2} \\
2 A \tilde{r}_{4}^{\mathrm{opt}}=\alpha \omega_{1}^{4}+\beta \omega_{2}^{4} \\
A=\alpha c^{4} \omega_{1}^{4}+2 \alpha c^{2} \omega_{1}^{2}+\alpha .
\end{gathered}
$$

Finally, a simple set of algebraic equations are provided to help the reader to calculate the $D$-optimal input excitation power spectrum, $\alpha, \beta, \omega_{1}, \omega_{2}$. Substituting $\beta=K \tilde{r}_{0}^{\mathrm{opt}}-\alpha$ from (33a) into (35b)-(35d), we have

$$
\begin{aligned}
& 2 A \tilde{r}_{2}^{\mathrm{opt}}=\alpha \omega_{1}^{2}+2 A \tilde{r}_{0}^{\mathrm{opt}} \omega_{2}^{2}-\alpha \omega_{2}^{2} \\
& 2 A \tilde{r}_{4}^{\mathrm{opt}}=\alpha \omega_{1}^{4}+2 A \tilde{r}_{0}^{\mathrm{opt}} \omega_{2}^{4}-\alpha \omega_{2}^{4} \\
& A=\alpha c^{4} \omega_{1}^{4}+2 \alpha c^{2} \omega_{1}^{2}+\alpha .
\end{aligned}
$$

From (36a), we find that $\omega_{2}^{2}=\left[2 A \tilde{r}_{2}^{\text {opt }}-\alpha \omega_{1}^{2}\right] /\left[2 A \tilde{r}_{0}^{\text {opt }}-\right.$ $\alpha]$, which gives after some calculations the $D$-optimal multisine excitation power spectrum, $\alpha, \beta, \omega_{1}, \omega_{2}$, namely:

(i) Given $A$, calculate $K$ :

$K=2 A$.

(ii) Determine the intermediate quantities $x, y$ and $z$ from the numerical values found in (25) and the impedance model parameter $c$ :

$$
\begin{aligned}
& x=2\left[\tilde{r}_{0}^{\mathrm{opt}} \tilde{r}_{4}^{\mathrm{opt}}-\left(\tilde{r}_{2}^{\mathrm{opt}}\right)^{2}\right] c^{4}-\tilde{r}_{0}^{\mathrm{opt}} \\
& y=2\left[\tilde{r}_{0}^{\mathrm{opt}} \tilde{r}_{4}^{\mathrm{opt}}-\left(\tilde{r}_{2}^{\mathrm{opt}}\right)^{2}\right] c^{2}+\tilde{r}_{2}^{\mathrm{opt}} \\
& z=2\left[\tilde{r}_{0}^{\mathrm{opt}} \tilde{r}_{4}^{\mathrm{opt}}-\left(\tilde{r}_{2}^{\mathrm{opt}}\right)^{2}\right]-\tilde{r}_{4}^{\mathrm{opt}} .
\end{aligned}
$$

(iii) Find the $D$-optimal multisine excitation power spectrum:

$$
\begin{aligned}
\omega_{1}^{2} & =\frac{-y \pm \sqrt{y^{2}-x z}}{x} \\
\alpha & =\frac{A}{\left(c^{2} \omega_{1}^{2}+1\right)^{2}} \\
\omega_{2}^{2} & =\frac{2 A \tilde{r}_{2}^{\mathrm{opt}}-\alpha \omega_{1}^{2}}{2 A \tilde{r}_{0}^{\mathrm{opt}}-\alpha} \\
\beta & =2 A \tilde{r}_{0}^{\mathrm{opt}}-\alpha .
\end{aligned}
$$

\section{Simulation results}

To validate the theory presented in sections 3.1 and 3.2, sections 4.1 and 4.2 simulate the $D$-optimal multisine excitation power spectrum as a function of the multisine excitation frequencies $\omega_{1}$ and $\omega_{2}$ and power spectrum amplitudes $\alpha$ and $\beta$ for the 2R-1C impedance model considered. Next, section 4.3 analyzes the variance of the impedance model parameters $\theta$ as a function of the excitation frequencies $\omega_{1}$ and $\omega_{2}$ considering the covariance matrix elements defined in (2). Later, section 4.4 analyzes the accuracy of the model parameter estimates from (8) from input current-noisy output voltage measurements.

For convenience, the definition of the $2 \mathrm{R}-1 \mathrm{C}$ impedance model is made in terms of $a, b$ and $c$ since, depending on the reader's interest, it is possible to find the circuit elements that fit best his/her needs (see (9) and (10)). The true model parameters considered are $a=0.1, b=1, c=0.1$. Unless otherwise stated, the frequency grid considered is linearly spaced from 1 to $30 \mathrm{rad} \mathrm{s}^{-1}$ (100 spectral points).

The $D$-criterion admits infinite solutions but only two of them are analyzed in this paper: the first, for an input signal with $\omega_{1}^{\text {opt }}=0$ (see (29)), can be found using (25), which gives $\omega_{2}^{\text {opt }}=17.3203 \mathrm{rad} \mathrm{s}^{-1}, \alpha=0.3333$ and $\beta=0.0417$. The second, which consists in assuming that $\omega_{1}^{\text {opt }}$ is non-zero but where the two sinusoids are forced to have equal amplitude ( $\alpha=\beta=A=0.5$ ), is studied in section 4.1.

\subsection{D-criterion of the Fisher information matrix}

In order to calculate the full Fisher information matrix, it can be noted that it corresponds to the sum of two components, corresponding to the excitation frequencies $\omega_{1}$ and $\omega_{2}$ of a multisine excitation (according to (17) and (26)), namely:

$$
\bar{M}\left(\omega_{1}, \omega_{2}\right)=\alpha M\left(\omega_{1}\right)+\beta M\left(\omega_{2}\right) .
$$

The plotting of the $D$-criterion for a 2-sine input of the determinant of (40) is somewhat more complicated since this time it depends on two frequencies instead of one as the example presented in section 2.1. Therefore, the determinant cannot be represented in two (see figure 1) but three dimensions (see figure 3), where the corresponding figure axes $x$ and $y$ refer to the multisine excitation frequencies $\omega_{1}$ and $\omega_{2}$, respectively, and the values in the $z$-axis correspond 


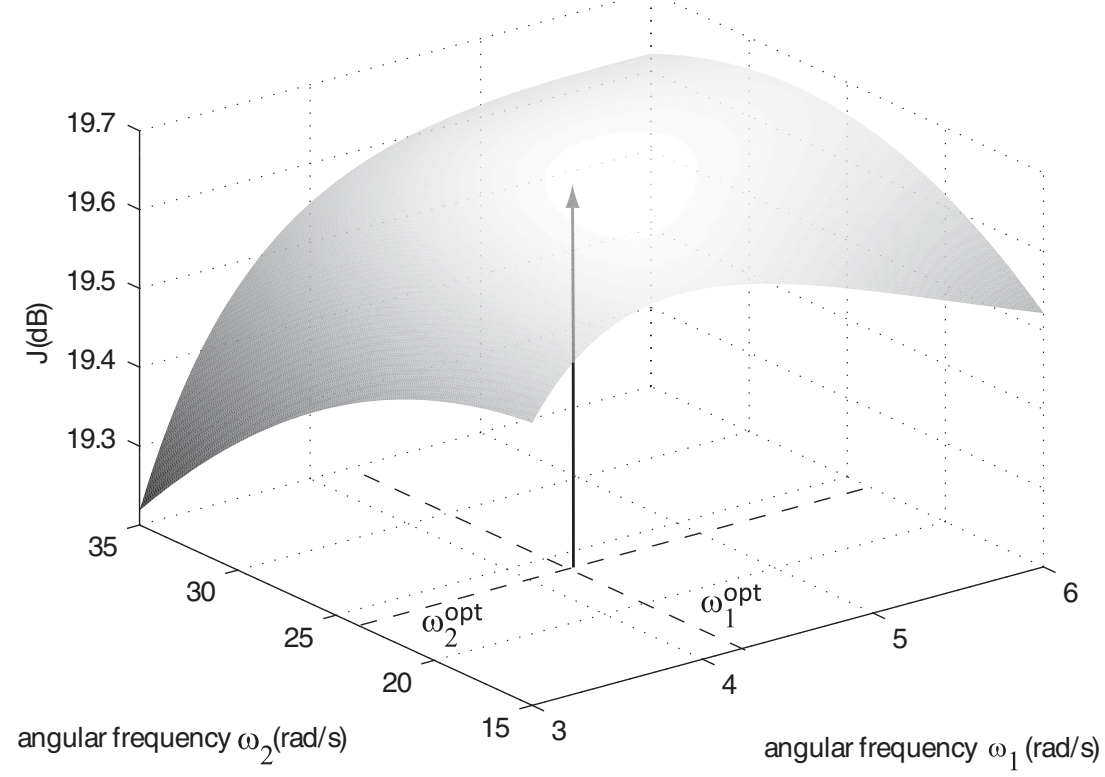

Figure 3. $D$-criterion surface plot of the full Fisher information matrix for the $2 \mathrm{R}-1 \mathrm{C}$ impedance model $Z(s)$ as a function of the multisine excitation frequencies $\omega_{1}$ and $\omega_{2}$. The maximum of $J$ (see (41)) matches the optimal excitation frequencies $\omega_{1}^{\mathrm{opt}}=4.1420 \mathrm{rad} \mathrm{s}^{-1}$ and $\omega_{2}^{\text {opt }}=24.1413 \mathrm{rad} \mathrm{s}^{-1}$ solution calculated using CVX.

(A) $\alpha=\beta=0.5$

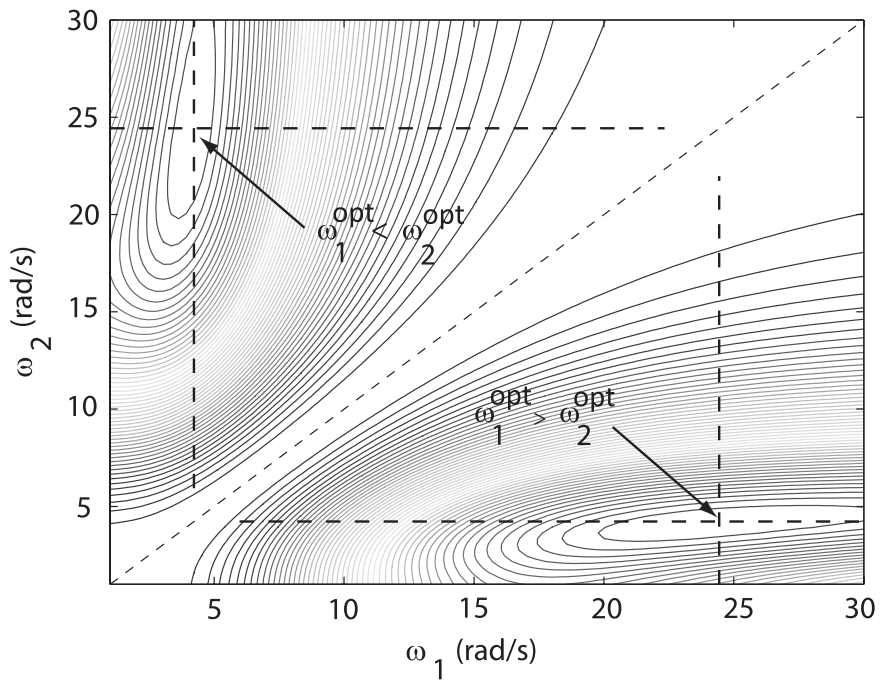

(B) $\alpha=0.5, \beta=0.4$

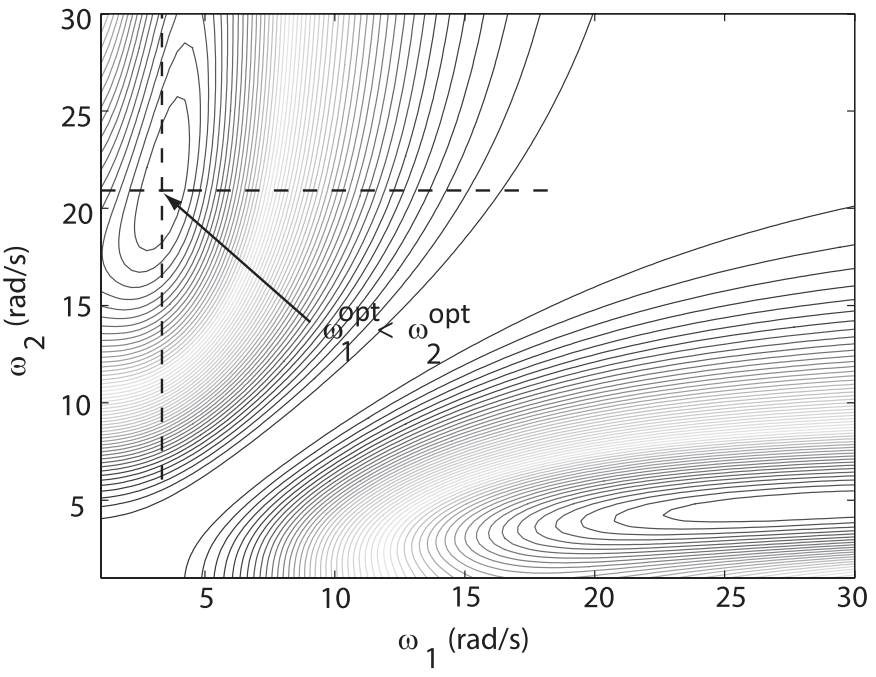

Figure 4. $D$-criterion contour plot of the full Fisher information matrix for the $2 \mathrm{R}-1 \mathrm{C}$ impedance model as a function of the multisine excitation frequencies $\omega_{1}$ and $\omega_{2}$. The intersection of the dotted lines indicates the maximum of the determinant, which is produced at the optimal multisine excitation frequencies $\omega_{1}^{\mathrm{opt}}=4.1420 \mathrm{rad} \mathrm{s}^{-1}$ and $\omega_{2}^{\mathrm{opt}}=24.1413 \mathrm{rad} \mathrm{s}^{-1}\left(\alpha=\beta=0.5, \omega_{1}^{\mathrm{opt}} \leqslant \omega_{2}^{\mathrm{opt}}, A\right)$ and $\omega_{1}^{\text {opt }}=3.3434 \mathrm{rad} \mathrm{s}^{-1}$ and $\omega_{2}^{\text {opt }}=20.9192 \mathrm{rad} \mathrm{s}^{-1}\left(\alpha=0.5, \beta=0.4, \omega_{1}^{\text {opt }} \leqslant \omega_{2}^{\text {opt }}, B\right)$. See the text for details.

to the determinant of the full Fisher information matrix. Although it is possible to visually identify the maximum of the determinant from a three-dimensional graph (see figure 3), it is preferable, however, to do so in two dimensions and to represent the same information by contour lines (see figures $4(A)$ and $4(B)$ ). Accordingly, areas of greater density of contour lines will be related to the excitation frequencies that obtain maximum information (maximum of the determinant) and, conversely, the areas of lower density of contour lines correspond to excitation frequencies with less information extracting capability.

The $D$-criterion solution for the excitation power spectrum $\Phi_{u}(\omega)$ is

$$
\left.\begin{array}{l}
J\left(\omega_{1}, \omega_{2}\right) \\
=\log \operatorname{det}
\end{array} \int_{0}^{\infty}\left[\begin{array}{ccc}
1 & 0 & -\omega^{2} \\
0 & \omega^{2} & 0 \\
-\omega^{2} & 0 & \omega^{4}
\end{array}\right] \frac{\Phi_{u}(\omega)}{\left(c^{2} \omega^{2}+1\right)^{2}} \mathrm{~d} \omega\right\}
$$


If $\Phi_{u}(\omega)$ is replaced by the power spectrum defined by 2 -sine input excitation, the $D$-criterion solution is

$$
\begin{aligned}
& J\left(\omega_{1}, \omega_{2}\right) \\
& =\log \operatorname{det}
\end{aligned}\left\{\sum_{i=1}^{2} \frac{1}{2\left(c^{2} \omega_{i}^{2}+1\right)^{2}}\left[\begin{array}{ccc}
1 & 0 & -\omega_{i}^{2} \\
0 & \omega_{i}^{2} & 0 \\
-\omega_{i}^{2} & 0 & \omega_{i}^{4}
\end{array}\right]\right\}
$$

and it can be simplified into

$$
\begin{aligned}
& J\left(\omega_{1}, \omega_{2}\right) \\
& \quad=\log [P]+\log \left[(B+C)\left(B \omega_{1}^{4}+C \omega_{2}^{4}\right)-P^{2}\right]-3 \log (2),
\end{aligned}
$$

where the quantities $B, C$ and $P$ are defined as

$$
\begin{aligned}
& B=\frac{1}{\left(c^{2} \omega_{1}^{2}+1\right)^{2}} \\
& C=\frac{1}{\left(c^{2} \omega_{2}^{2}+1\right)^{2}} \\
& P=B \omega_{1}^{2}+C \omega_{2}^{2} .
\end{aligned}
$$

Figure 3 illustrates the $D$-criterion given by (41) as a function of the excitation frequencies $\omega_{1}$ and $\omega_{2}$. This plot shows that the maximum of $J$ seems to be achieved close to the axes, i.e. when either $\omega_{1}$ or $\omega_{2}$ (but not both) is very close to 0 . In fact, when $\omega_{1}=\omega_{2}, J$ becomes $-\infty$, due to the lack of persistence of excitation. The same happens if $\omega_{1}$ and $\omega_{2}$ are very close to each other. This means the following: it is not possible to accurately estimate the impedance model parameters vector $\theta^{*}$ from a 2 -sine input excitation unless the excitation frequencies $\omega_{1}$ and $\omega_{2}$ are sufficiently far from each other.

Using the procedure described and considering the values given in (25), we obtain that the $D$-optimal excitation spectrum is given by the excitation frequencies $\omega_{1}^{\text {opt }}=4.1420 \mathrm{rad} \mathrm{s}^{-1}$ and $\omega_{2}^{\mathrm{opt}}=24.1413 \mathrm{rad} \mathrm{s}^{-1}$ (where we also impose $0 \leqslant \omega_{1} \leqslant$ $\omega_{2}$, since otherwise we would obtain eight possible sets of solutions). To corroborate this, figure $4(A)$ shows the contour lines of $J$ in a neighborhood of the maximum where the dotted lines indicates the $D$-criterion optimal solution found with CVX. The diagonal symmetry in figure $4(A)$ is due to the fact that the frequency grids considered for both excitation frequencies are the same, with the same excitation power spectrum amplitude $\alpha=\beta=0.5$. It can be observed that the maximum of the determinant is produced at two specific frequencies, due to the symmetry reasons described above.

However, such diagonal symmetry is lost in figure $4(B)$, since now the excitation power spectrum amplitudes $\alpha$ and $\beta$ are no longer the same. Assuming that the amplitude of each of the components $\alpha$ and $\beta$ is different, the set of frequencies where the maximum of the full Fisher information matrix determinant occurs changes with respect the results shown in figure 4(A). It makes sense since we fixed that $\beta<\alpha$, which means that those frequencies with a greater amplitude power spectrum will contribute to obtain more information on the impedance. Nevertheless, if we sweep the values $\alpha$ and $\beta$, then the resultant plot will be symmetrical to figure $4(B)$.
Table 1. Optimal multisine excitation frequencies $\omega_{1}^{\text {opt }}$ and $\omega_{2}^{\text {opt }}$ depending on the excitation power spectrum amplitude $\beta$ (see figure 6).

\begin{tabular}{lcrcc}
\hline & \multicolumn{4}{c}{$\alpha=0.5$} \\
\cline { 2 - 5 } & $\beta_{1}=0.5$ & $\beta_{2}=0.4$ & $\beta_{3}=0.3$ & $\beta_{4}=0.2$ \\
\hline$\omega_{1}^{\text {opt }}\left(\mathrm{rad} \mathrm{s}^{-1}\right)$ & 4.14 & 3.34 & 2.17 & 1 \\
$\omega_{2}^{\text {opt }}\left(\mathrm{rad} \mathrm{s}^{-1}\right)$ & 24.14 & 20.92 & 18.58 & 17.40 \\
\hline
\end{tabular}

\subsection{Dependence of the D-optimal multisine excitation frequencies $\omega_{1}^{\mathrm{opt}}$ and $\omega_{2}^{\mathrm{opt}}$ on $\theta, \alpha$ and $\beta$}

To illustrate the position of optimal excitation frequencies over the impedance spectrum, several impedance frequency responses according to (8) have been simulated (see figure 5) for different values of $b$ and considering $a=0.1$ and $c=0.1$. The position of the optimal excitation frequencies $\omega_{1}^{\text {opt }}$ and $\omega_{2}^{\text {opt }}$ corroborates the fact that the $D$-optimal excitation power spectrum only depends on the parameter $c$ but not on $b$ or $a$; that is the reason why they are fixed close to the pole-zero cutoff frequencies.

Table 1 reports the values for the optimal multisine frequencies $\omega_{1}^{\text {opt }}$ and $\omega_{2}^{\text {opt }}$ as a function of the excitation power spectrum amplitude $\beta$ ( $\alpha$ has been assumed to be equal to 0.5 ). The same information is plotted in figure 6 over the simulated impedance magnitude spectrum. It can be observed in the figure how the fact that the spectral line $\alpha$, related to $\omega_{1}$, applies more energy into the system than the spectral line $\beta$, related to $\omega_{2}$, makes the optimal excitation frequency $\omega_{1}^{\text {opt }}$ move fast toward the lower frequencies placed at the $x$-axis since it becomes more important $\left(\omega_{1}^{\text {opt }}\right.$ provides more impedance information than $\omega_{2}^{\mathrm{opt}}$ ). At the same time, the optimal excitation $\omega_{2}^{\text {opt }}$ moves toward lower frequencies until a certain value beyond which there is no significant displacement. The explanation why this happens is that, given a certain value of $\beta$, the optimal excitation $\omega_{2}^{\text {opt }}$ must provide information on the position of the impedance model parameter $a / c$ and this occurs at high frequencies. Hence, $\omega_{2}^{\text {opt }}$ does not shift to frequencies below a certain limit.

Finally, figure 7 shows how the optimal frequencies $\omega_{1}^{\text {opt }}$ and $\omega_{2}^{\text {opt }}$ depend on the impedance model parameter $c$. It can be observed that the frequency values for the optimal increase as the value $c$ increases, moving toward higher frequencies.

The reader should be aware that the standard approach of most excitation optimization algorithms is to maximize the determinant of the full Fisher information matrix, instead of working with the covariance matrix of the actual estimators being used (which coincide only asymptotically, for asymptotically efficient estimators). In practice, most of the optimization approaches just calculate the Fisher information matrix and work with its determinant, since its calculation is much easier than the (finite sample) covariance matrix itself.

As mentioned in the text, the $D$-criterion is a generalpurpose criterion which cannot particularly be the best choice for a particular impedance measurement application (since it takes into account all the Fisher information matrix elements for the excitation optimization). For that reason, the optimal excitation frequencies $\omega_{1}^{\text {opt }}$ and $\omega_{2}^{\text {opt }}$ are situated so the 

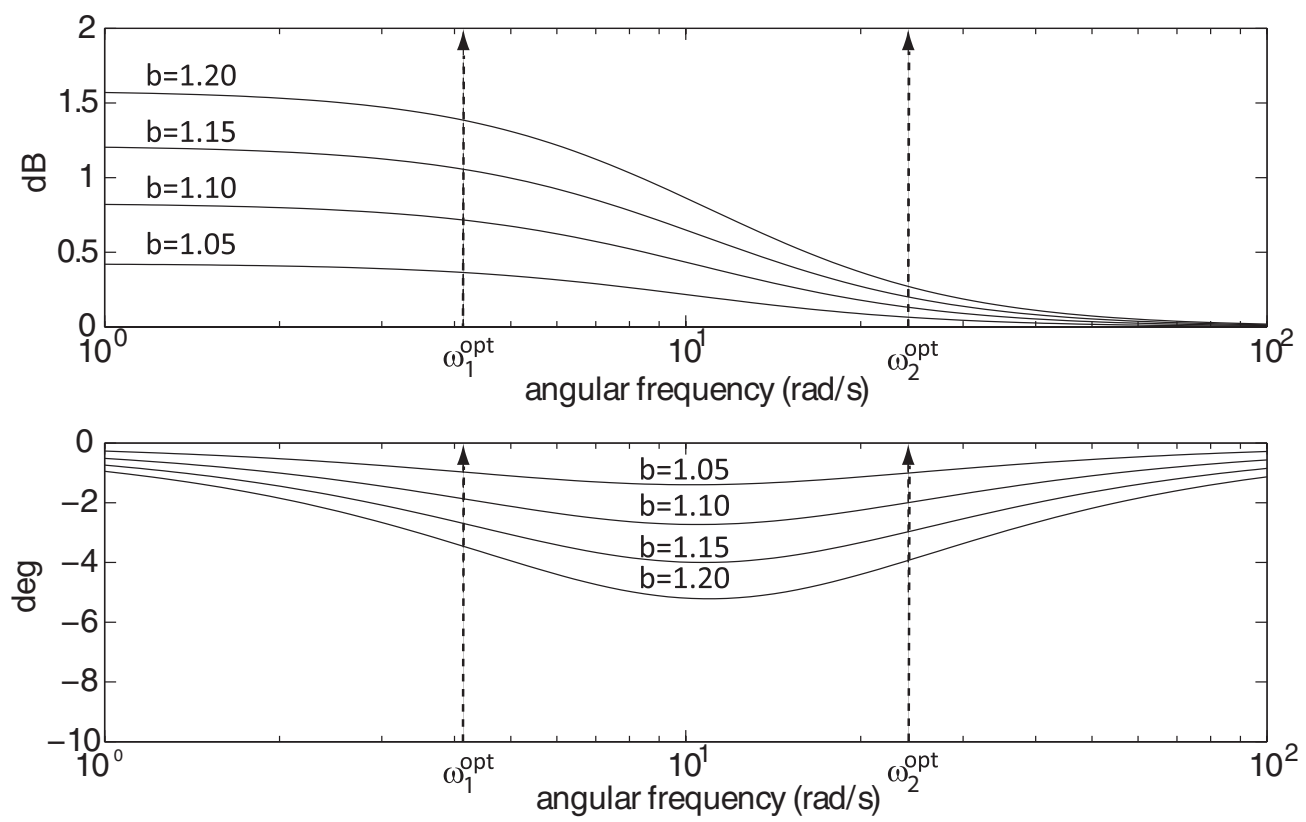

Figure 5. Magnitude (top, units in decibels) and phase (bottom) of the simulated impedance frequency response. The vertical dotted arrows indicate the fixed position of the optimal excitation frequencies $\omega_{1}^{\mathrm{opt}}=4.1420 \mathrm{rad} \mathrm{s}^{-1}$ and $\omega_{2}^{\mathrm{opt}}=24.1413 \mathrm{rad} \mathrm{s}^{-1}$ (see figure 4), which are independent of the impedance frequency response being simulated. The $b$ values simulated were $b=1.05,1.10,1.15,1.20(a=0.1$, $c=0.1, \alpha=\beta=0.5$ ).

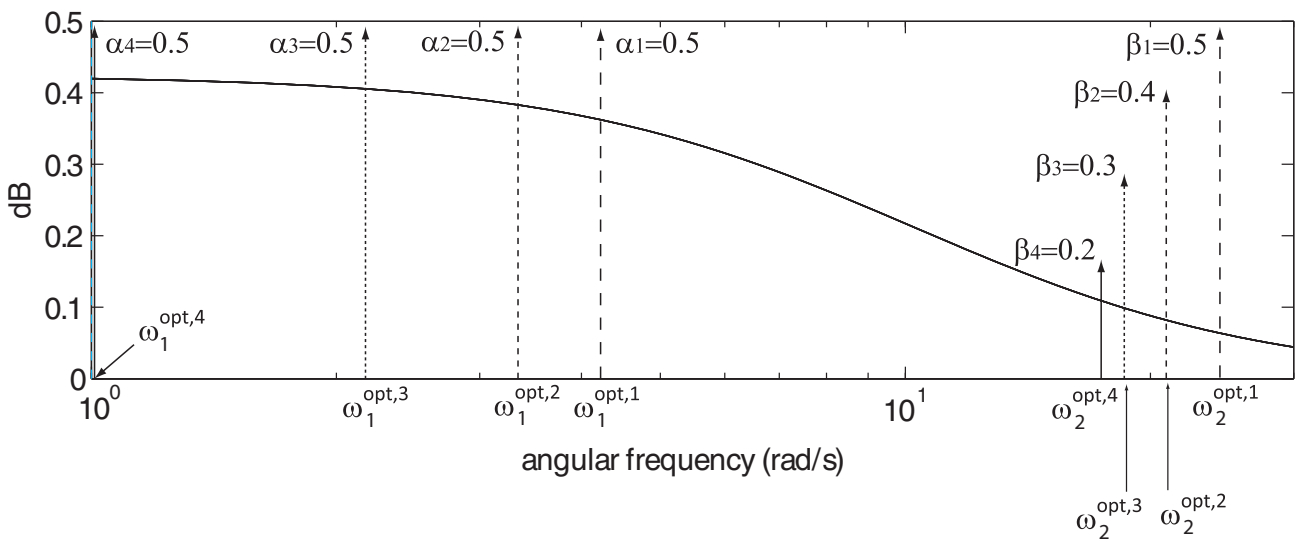

Figure 6. Magnitude (units in decibels) of the simulated impedance frequency response. The vertical dotted arrows illustrate the amplitude spectrum $\alpha$ and $\beta$ for the optimal multisine spectral lines at the excitation frequencies $\omega_{1}^{\text {opt }}$ and $\omega_{2}^{\text {opt }}$, respectively (the numerical values for $\omega_{1}^{\mathrm{opt}}$ and $\omega_{2}^{\mathrm{opt}}$ are given in table 1) $(a=0.1, b=1.05, c=0.1)$.

maximum information is gained from the experiment to estimate all the impedance model parameters $\theta$. Instead, it is sometimes preferable to have knowledge of the variance of each impedance model parameter as a function of frequency separately, like the example shown in figure 1, in order to distribute the excitation frequencies, i.e. the multisine frequency grid, optimally for a given function of the parameter vector. If, for example, one is interested only in estimating $a$ for the example shown in section 2.1, a more suitable criterion would be to minimize its variance, $\sigma_{a}^{2}$, defined as the first element of the covariance matrix, namely:

$\sigma_{a}^{2}(\omega)=\left[M(\omega)^{-1}\right]_{11}=\left[\int_{-\infty}^{\infty}\left(a^{2}+b^{2} \omega^{2}\right)^{-2} \Phi_{u}(\omega) \mathrm{d} \omega\right]^{-1}$ which, for an input power budget constraint, e.g.,

$$
\int_{-\infty}^{\infty} \Phi_{u}(\omega) \mathrm{d} \omega \leqslant 1
$$

it is minimized by a sinusoid of frequency $\omega^{*}$ and amplitude 0.5. Section 4.3 deals with the analysis of the variance of each of the parameters as a function of the multisine excitation frequencies, that is, using the covariance matrix elements calculated given by (40).

\subsection{Analysis of the covariance matrix}

Next, the variance of each impedance model parameter is studied as a function of the excitation frequencies $\omega_{1}$ and $\omega_{2}$ by the interpretation of the contour lines defined by the diagonal elements of the covariance matrix. The parameters 

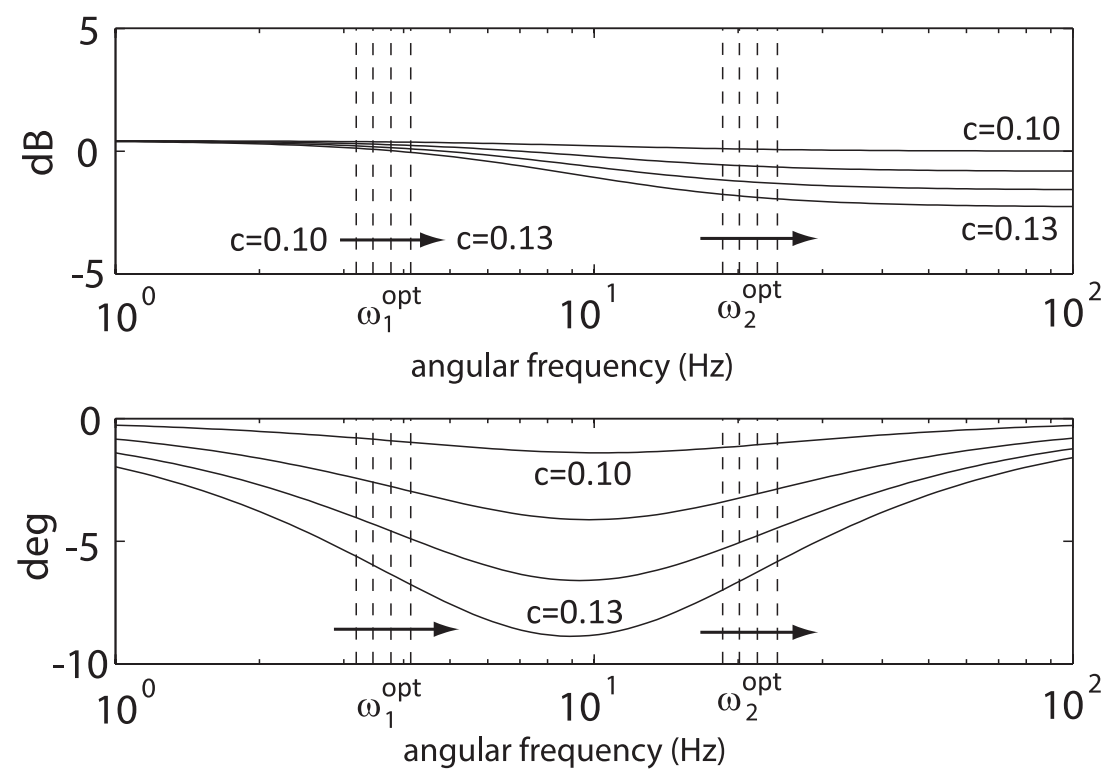

Figure 7. Magnitude (top, units in decibels) and phase (bottom) of the simulated impedance frequency response. The vertical dotted lines represent the position of the optimal excitation frequencies $\omega_{1}^{\mathrm{opt}}$ and $\omega_{2}^{\mathrm{opt}}$ as a function of the values of the impedance model parameter $c$ $(c=0.1,0.11,0.12,0.13)$. The arrows indicate the movement direction of the optimal excitation frequencies $(a=0.1, b=1.05$, $\alpha=\beta=0.5)$. The numerical values for $\omega_{1}^{\mathrm{opt}}$ and $\omega_{2}^{\mathrm{opt}}$ as a function of $c$ are not shown.
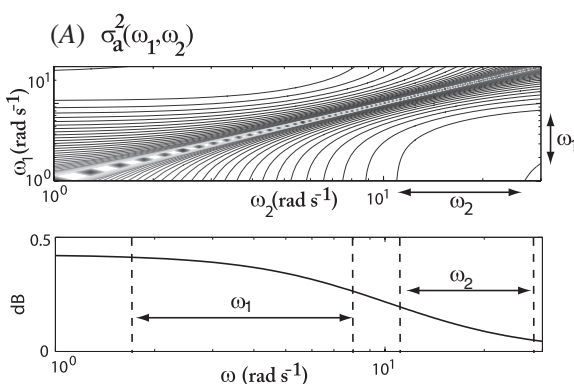

$$
\text { (B) } \sigma_{b}^{2}\left(\omega_{1}, \omega_{2}\right)
$$
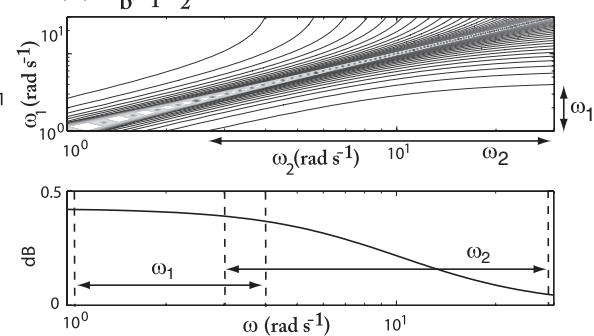

(C) $\sigma_{\mathrm{c}}^{2}\left(\omega_{1}, \omega_{2}\right)$
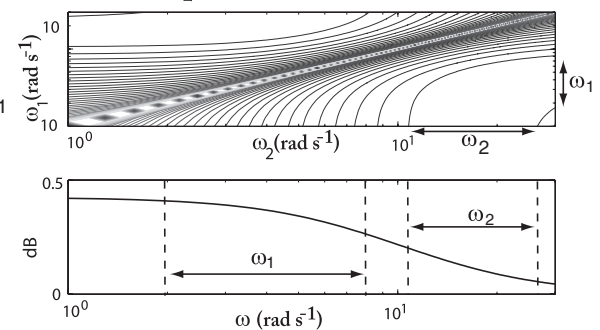

Figure 8. Frequency dependence of the variance of the impedance model parameters $\theta, \sigma_{a}^{2}\left(\omega_{1}, \omega_{2}\right)(A), \sigma_{b}^{2}\left(\omega_{1}, \omega_{2}\right)(B)$ and $\sigma_{c}^{2}\left(\omega_{1}, \omega_{2}\right)(C)$. The double-headed arrows indicate the frequency ranges where the isovariance lines are further apart; and its implication for the optimal frequency range for allocating the excitation frequencies $\omega_{1}$ and $\omega_{2}$ according to the impedance model simulated (bottom, units in decibels).

considered for the simulations are $a=0.1, b=1.05, c=0.1$, $\alpha=\beta=0.5$. It can be seen from figures $8(A)-(C)$ (top) that the optimal excitation frequency ranges to accurately measure $a, b$ and $c$ (this is with minimum variance of the parameters $\sigma_{a}^{2}\left(\omega_{1}, \omega_{2}\right)=\left[M^{-1}\right]_{11}, \sigma_{b}^{2}\left(\omega_{1}, \omega_{2}\right)=\left[M^{-1}\right]_{22}$ and $\left.\sigma_{c}^{2}\left(\omega_{1}, \omega_{2}\right)=\left[M^{-1}\right]_{33}\right)$ are contained in areas where the so-called isovariance lines are further apart. In order to help the reader to interpret the data shown by the isovariance lines, the double-headed arrows indicate the $\omega_{1}$ and $\omega_{2}$ frequency ranges where the isovariance lines are further apart, plotted over the impedance spectrum magnitude Bode diagram in vertical lines. Figures $8(A)-(C)$ (bottom) show how the spread of the isovariance lines can be used as a design guide for the selection of the excited frequency range. In order to obtain the best possible estimate for the parameter $a$ (minimum variance), one excitation frequency, e.g. $\omega_{2}$, must be above the cutoff frequency defined by the zero of the transfer function $Z(s)$. As for the frequency $\omega_{1}$, it may be between low frequencies and the cutoff frequency defined by the pole of the impedance model $Z(s)$ (see figure $8(A)$ ).
To $D$-optimally estimate the parameter $b$, one excitation frequency, e.g. $\omega_{1}$, must be below the cutoff frequency defined by the pole of the impedance transfer function $Z(s)$ (see figure $8(B)$ ). As for the frequency range for $\omega_{2}$, it may be arbitrarily placed as long as both frequencies are sufficiently far apart. Otherwise, the full Fisher information matrix would be ill conditioned, and the elements from the covariance matrix would tend to infinity. This means that for close excitation frequencies $\omega_{1}$ and $\omega_{2}$, there is insufficient information to estimate this parameter. By analogy with the circuit shown in figure 2 , one would expect that since the variance of $b$ directly corresponds to the variance of the parameter $R_{e}$, measuring it at low frequencies would be the best option.

The case for the optimal estimate of the parameter $c$ shown in figure $8(C)$ is qualitatively identical to the situation for $a$.

\subsection{Identification of the impedance model from input current-noisy voltage output measurements}

At this point of this paper, the $D$-optimal multisine excitation spectrum has been designed in section 3 and validated through 
Table 2. Estimated impedance model parameters $\widehat{\theta}$ from the simulated input current-noisy voltage output measurements. The true values for the impedance model are $a=0.1, b=1.05$ and $c=0.1$.

\begin{tabular}{llll}
\hline & $\widehat{a}$ & $\widehat{b}$ & $\widehat{c}$ \\
\hline$\omega_{1}^{\mathrm{opt}}, \omega_{2}^{\mathrm{opt}}$ & 0.0889 & 1.0524 & 0.0882 \\
$\omega_{1}, \omega_{2}$ & 0.1637 & 1.0601 & 0.1621 \\
\hline
\end{tabular}

simulations in section 4 . Thus, the last thing pending to be done is to corroborate that, by using the $D$-optimal spectrum, it is possible to identify the impedance model parameters. To do this, we simulated the impedance model simulated in section $4(a=0.1, b=1.05$ and $c=0.1)$ using the MATLAB command filter which generates a continuoustime equivalent of the impedance system. The multisine input excitation is normalized so that its rms value is 1 . One case is considered with white Gaussian additive noise at the output, where its standard deviation is ten times smaller than the rms of the output signal. The impedance transfer function has been determined using the MATLAB frequency domain system identification toolbox. The experiment is repeated 100 times with a fixed input excitation spectrum (considering random phases uniformly distributed in $[0,2 \pi)$ ) and a different noise realization for each repetition. From the time current and voltage signals, a parametric impedance model has been calculated using the elis routine. The impedance frequency response and its variance are estimated at the multisine excitation frequencies, $\omega_{1}^{\mathrm{opt}}=4.14 \mathrm{rad} \mathrm{s}^{-1}$, $\omega_{2}^{\text {opt }}=24.14 \mathrm{rad} \mathrm{s}^{-1}$, and at two other non-optimal excitation frequencies $\omega_{1}=3 \mathrm{rad} \mathrm{s}^{-1}, \omega_{2}=15 \mathrm{rad} \mathrm{s}^{-1}$ (the amplitude power spectrum was the same for both cases, $\alpha=\beta=0.5$ ). It can be seen from table 2 that the $D$-optimal power spectrum gets a better estimate of model parameters compared to a non-optimal power spectrum. In general and for all the possible cases, it is not true that the $D$-optimal excitation gives smaller variance for each parameter separately. Nevertheless and for the simulation performed, the results confirm that the $D$-optimal excitation power spectrum gives a more reliable estimate of each individual parameter.

\section{Experimental measurements}

To experimentally validate the methodology described in section 3 and simulated in section 4 , we measured the impedance frequency response of a $2 \mathrm{R}-1 \mathrm{C}$ electrical circuit like the one shown in figure 2 (top) using a customized multi-frequency impedance analyzer built around a rugged PCplatform based on a PXI (PCI eXtensions for Instrumentation) system from National Instruments. The customized impedance analyzer system includes an embedded controller PXIe8130, a two-channel high-speed digitizer card PXIe-5122 (100 $\mathrm{Ms} \mathrm{s}^{-1}, 64 \mathrm{MB} / \mathrm{channel,} 14$ bits) and an arbitrary waveform generator (AWG) card PXI-5422 (200 $\mathrm{Ms} \mathrm{s}^{-1}$, $32 \mathrm{MB}, 16$ bits). Synchronously to the excitation multisine $r(t)$ generated by the AWG PXI-5422, the two-channel digitizer PXIe-5122 simultaneously acquired the voltage $v(t)$ and the current $i(t)$.
Table 3. Estimated impedance model parameters $\widehat{\theta}$ from current and voltage measurements. The true values are $a=6.12 \times 10^{-4}$, $b=510$ and $c=6.3 \times 10^{-6}$.

\begin{tabular}{llll}
\hline & $\widehat{a}$ & $\widehat{b}$ & $\widehat{c}$ \\
\hline$\omega_{1}^{\mathrm{opt}}, \omega_{2}^{\mathrm{opt}}$ & $6.82 \times 10^{-4}$ & 489.16 & $7.36 \times 10^{-6}$ \\
$\omega_{1}, \omega_{2}$ & $6.86 \times 10^{-4}$ & 486.71 & $7.44 \times 10^{-6}$ \\
\hline
\end{tabular}

The impedance spectrum was calculated as the mean impedance magnitude spectrum determined at the excitation frequency $k$ by applying the classical spectral analysis based on cross and auto power spectrum using a rectangular window [28]. Four steady-state periods of the current $i(t)$ and voltage $v(t)$ signals were sampled at $20 \mathrm{MHz}$.

Due to limitations on the frequency bandwidth of the measuring equipment, it was not possible to measure the same impedance model frequency response considered in the simulations. The reason is that this impedance model has the relaxation at frequencies out of the frequency bandwidth of the measuring equipment. Instead, the experimental measurement was carried out considering the same impedance equivalent 2R-1C circuit but with different values of impedance model parameters, i.e. $a=6.12 \times 10^{-4}, b=510$ and $c=6.3 \times 10^{-6}$. For this case, the $D$-optimal excitation frequencies are located at $\omega_{1}^{\mathrm{opt}}=6.7 \times 10^{4} \mathrm{rad} \mathrm{s}^{-1}$ and $\omega_{2}^{\mathrm{opt}}=$ $3.8 \times 10^{5} \mathrm{rad} \mathrm{s}^{-1}$. The excitation frequencies considered for the non-optimal power spectrum were $\omega_{1}=1.9 \times 10^{5} \mathrm{rad} \mathrm{s}^{-1}$ and $\omega_{2}=3.14 \times 10^{5} \mathrm{rad} \mathrm{s}^{-1}$ (again $\alpha=\beta=0.5$ for both excitations). The results reported in table 3 are similar to the simulation results shown in table 2 . The estimation of the impedance model parameters using the $D$-optimal excitation power spectrum is more accurate than the nonoptimal excitation power spectrum.

\section{Discussion}

The option to construct the $D$-optimal input signal as a constant plus a sinusoidal would help to simplify the mathematical analysis. However, there are main practical limitations regarding biological measurements. Firstly, it is advisable (and sometimes compulsory), not to measure low frequency signals or dc signals. The reason is that low frequencies can stimulate organs and cause, for example, arrhythmias and fibrillation, among other complications. Secondly, the measurement at these low frequencies is not always possible in practice due to the high skin impedance at dc, for example, in body fluid measurements [47]. Thirdly, in applications for the determination of cell concentration in cell culture biotechnology applications [48, 49], applying a dc voltage is undesirable because it might cause electrolysis, which changes the $\mathrm{pH}$ of the culture medium and may kill cells. Nevertheless, in electrochemical spectroscopy, applying dc voltage is a common situation, usually to polarize the electrode; hence, it is superimposed on the signal for measuring the electrochemical impedance spectrum.

As explained through the text, the minimum number of excitation frequencies to fit impedance data into a $2 \mathrm{R}-1 \mathrm{C}$ impedance model is two. However, in practice, most 
of the commercial impedance analyzers use a larger number of excitation frequencies to fit the impedance data usually into more complex models, e.g. the Cole [50] or Randles [39] impedance models, which introduce semi-empirical elements such as constant phase elements (CPE) or diffusion like the Warburg element. Unlike the equivalent electrical $2 \mathrm{R}-1 \mathrm{C}$ circuit considered in this paper, the physical meaning of these semi-empirical elements is in many cases not clear and it depends on poorly predictable factors. Furthermore, by considering a $2 \mathrm{R}-1 \mathrm{C}$ impedance model, the $D$-optimal analysis becomes feasible and gives as a result an analytical solution. Nevertheless, impedance models including CPE or Warburg elements would require iterative algorithms due to their complexity hindering its interpretation and, at the end, these cases can be explained by extrapolating the results presented in this paper since the impedance frequency responses are similar within a frequency range of interest.

To the best of our knowledge, the first attempts in the literature addressing the influence of the excitation number of frequencies for bio-impedance spectroscopy measurements were proposed in $[51,52]$. The aim in both cases was similar: briefly, to determine the minimum number of excitation frequencies that are necessary, given a certain level of uncertainty in the estimation of the model parameters, to fit impedance data to a model. In other words, this is the same as to determine the level of accuracy on the estimation of the bio-impedance spectrum. In order to answer this question not only the number of frequencies used must be taken into account but, at least, also (1) how are these frequencies distributed along the impedance spectrum and (2) how the excitation power spectrum amplitude is designed. Related to the first aspect, the optimal frequency distribution for EBI spectroscopy measurements was presented by Sanchez et al in [40]. The authors demonstrated that the optimal frequency distribution focuses the excitation frequencies close to the characteristic frequency of the impedance relaxation. Therefore, fewer excited frequencies would be required using this frequency distribution compared to equidistant or logarithmic frequency distributions in order to obtain the same impedance information.

With regard to the aspect related to the multisine excitation power spectrum amplitude, Popkirov and Schindler in [18, 19] and later Sanchez et al in [53] demonstrated that the impedance spectrum accuracy is sensitive to how well or badly the excitation amplitude spectrum is distributed along the frequency. The results shown in this paper enable the reader to go a step further. It can be observed from the determinant of the full Fisher information matrix shown in figure 4 or from the impedance frequency response shown in figures 5 and 6 that, depending on how the excitation amplitude spectrum is designed, the optimal measuring frequencies change. Moreover, these optimal excitation frequencies also change as a function of the impedance model considered for the optimization (see figure 7). Furthermore, the optimal measuring frequency range depends on whether the aim is to individually measure with high accuracy any of the parameters of the impedance model (see figure 8) or all at once (see figure $4(A)$ ). For all the previously mentioned reasons, we can conclude that, in general, no unique solution exists which is optimal for all the possible impedance models.

Additionally, other experimental conditions must be taken into account, for example, the excitation features [54, 55, 28], the influence of the electrode impedance [56], the properties of the system under investigation [57], the kind of excitation used (it is not the same to measure with a sinusoidal signal as using a (non-)periodic broadband signal [58]), and finally, the (non-)parametric time [59] or frequency [25] domain signal processing tools used to estimate the frequency response of a (non)linear impedance system [60-63].

\section{Conclusions}

The $D$-criterion is quite standard in the field of optimal experiment design, due to its interpretation (it is related to the volume of the confidence ellipsoids described by the Fisher information matrix) and parametrization independence (the $D$-optimal input does not change if the model is reparametrized). For the $2 \mathrm{R}-1 \mathrm{C}$ impedance model considered, the Fisher information matrix has a dimension $3 \times 3$, since three parameters need to be estimated. This means that, in order to obtain a non-singular full Fisher information matrix, a (multisine) signal with at least two non-negative excitation frequencies is required. In other words, the minimum number of excitation frequencies required to fit impedance data to a $2 \mathrm{R}-1 \mathrm{C}$ equivalent electrical model is two. This suggests a range of possibilities for input signals with different estimation properties but there is not a unique solution to optimally measure all cases. This implies that the input signal could be, e.g., a constant function plus a sinusoid, or two sinusoids. The optimal allocation of these frequencies depends on quantities such as the model parameters to be estimated. For those applications where the complexity of the impedance model considered to describe the data is higher, the $D$-optimal methodology is still valid and enables the design of the $D$-optimal excitation spectrum as well as the evaluation of the influence of the excitation spectrum (amplitude and frequency) on the variance of the model parameters.

It is finally important to remark that, instead of designing the optimal excitation power spectrum using the minimum number of frequencies required to fit data to an impedance model, an excitation including a larger number of frequencies (or even a continuous spectrum) would yield a more robust design in the presence of impedance model variations due to unknown phenomena not included in the optimization framework. Moreover, a signal with a large number of excited lines can be easily optimized in time by the minimization of its crest factor.

Summarizing, this paper has solved the $D$-criterion for the optimal multisine excitation power spectrum when measuring 2R-1C impedance models. For the first time, the influence of the excitation frequency distribution and power spectrum amplitude on the accuracy of the impedance model parameters has been analytically solved based on the experiment design theory. Furthermore, the optimal measuring frequency range for minimizing the variance of the impedance model parameters has been presented. Future 
work consists in performing a robust experiment design to evaluate the excitation robustness in the face of impedance system variations.

\section{Acknowledgments}

The authors would like to thank the anonymous reviewers for their comments on the manuscript. They are also grateful to Professor Hermann Scharfetter from the Institute of Medical Engineering at Graz University of Technology, Graz (Austria), for inspiring part of the technical content shown. This work has been supported in part by the Spanish Ministry SAF2011-30067-C02-02, by Redes de Investigacion del Instituto Carlos III (REDINSCOR, RD06/0003) and Fondo Europeo de Desarrollo Regional (FEDER), by the Swedish Research Council under contract 621-2011-5890, by the European Research Council under the advanced grant LEARN (contract 267381), by the Fund for Scientific Research (FWOVlaanderen), by the Flemish Government (Methusalem), and by the Belgian Government through the Interuniversity Poles of Attraction (IAP VI/4) Program.

\section{References}

[1] Gabriel S, Lau R W and Gabriel C 1996 The dielectric properties of biological tissues: II. Measurements in the frequency range $10 \mathrm{~Hz}$ to $20 \mathrm{GHz}$ Phys. Med. Biol. $412251-69$

[2] Casas O, Bragos R, Riu P, Rosell J, Tresanchez M, Warren M, Rodriguez A, Carreno A and Cinca J 1999

In vivo and in situ ischemic tissue characterization using electrical impedance spectroscopy Ann. NY Acad. Sci. 873 51-8

[3] Salazar Y, Bragos R, Casas O, Cinca J and Rosell J 2004 Transmural versus nontransmural in situ electrical impedance spectrum for healthy, ischemic, and healed myocardium IEEE Trans. Biomed. Eng. 51 1421-7

[4] Mellert F, Winkler K, Schneider C, Dudykevych T, Welz A, Osypka M, Gersing E and Preusse C 2010 Detection of (reversible) myocardial ischemic injury by means of electrical bioimpedance IEEE Trans. Biomed Eng. 58 1511-8

[5] Guan J-G, Miao Y-Q and Zhang Q-J 2004 Impedimetric biosensors J. Biosci. Bioeng. 97 219-26

[6] Katz E and Willner I 2003 Probing biomolecular interactions at conductive and semiconductive surfaces by impedance spectroscopy: routes to impedimetric immunosensors, DNA-sensors, and enzyme biosensors Electroanalysis 15 913-47

[7] Keay R and McNeil C 1998 Separation-free electrochemical immunosensor for rapid determination of atrazine Biosens. Bioelectron. 13 963-70

[8] Murphy L 2006 Biosensors and bioelectrochemistry Curr. Opin. Chem. Biol. 10 177-84

[9] Tudorache M and Bala C 2007 Biosensors based on screen-printing technology, and their applications in environmental and food analysis Anal. Bioanal. Chem. 388 565-78

[10] Orazem M E and Tribollet B 2008 Electrochemical Impedance Spectroscopy (Hoboken, NJ: Wiley)

[11] Godfrey K R 1993 Perturbation Signals for System Identification (Hemel Hempstead, UK: Prentice Hall International)

[12] Creason S and Smith D 1972 Fourier transform faradaic admittance measurements II. Ultra-rapid, high precision acquisition of the frequency response profile J. Electroanal. Chem. 40 A1-5

[13] Creason S, Hayes J W and Smith D E 1973 Fourier transform faradaic admittance measurements III. Comparison of measurement efficiency for various test signal waveforms J. Electroanal. Chem. Interfacial Electrochem. 47 9-46

[14] Schwall R J, Bond A M and Smith D E 1977 On-line fast Fourier transform faradaic admittance measurements: real-time deconvolution of heterogeneous charge transfer kinetic effects for thermodynamic and analytical measurements Anal. Chem. 49 1805-12

[15] Ichise M, Nagayanagi Y and Kojima T 1974 Application of pseudo-random signals and cross-correlation techniques in electroanalytical chemistry J. Electroanal. Chem. 49 187-98

[16] Pintelon R and Schoukens J 2012 System Identification: A Frequency Domain Approach (Piscataway, NJ: IEEE Press)

[17] Gabrielli C, Huet F and Keddam M 1992 Comparison of sine wave and white noise analysis for electrochemical impedance measurements J. Electroanal. Chem. 335 33-53

[18] Popkirov G S and Schindler F 1993 Optimization spectroscopy of the perturbation signal for electrochemical in the time domain impedance Rev. Sci. Instrum. $643111-5$

[19] Popkirov G S and Schlinder R 1994 The perturbation signal for fast Fourier transform electrochemical impedance spectroscopy (FFT-EIS) Bulg. Chem. Commun. 27 459-67

[20] Darowicki K, Orlikowski J and Lentka G 2000 Instantaneous impedance spectra of a non-stationary model electrical system J. Electroanal. Chem. 486 106-10

[21] Van Ingelgem Y, Tourwé E, Blajiev O, Pintelon R and Hubin A 2009 Advantages of odd random phase multisine electrochemical impedance measurements Electroanalysis 21 730-9

[22] Breugelmans T, Tourwé E, Jorcin J-B, Alvarez Pampliega A, Geboes B, Terryn H and Hubin A 2010 Odd random phase multisine EIS for organic coating analysis Prog. Org. Coat. $69215-8$

[23] Wilson A J, Milnes P, Waterworth A R, Smallwood R H and Brown B H $2001 \mathrm{Mk3}$.5: a modular, multi-frequency successor to the Mk3a EIS/EIT system Physiol. Meas. 22 49-54

[24] Bragos R, Blanco-Enrich R, Casas O and Rosell J 2001 Characterisation of dynamic biologic systems using multisine based impedance spectroscopy IEEE Proc. Instrumentation and Measurement Technology Conf. vol 1, pp 44-7

[25] Sanchez B, Schoukens J, Bragos R and Vandersteen G 2011 Novel estimation of the electrical bioimpedance using the local polynomial method. Application to in-vivo real-time myocardium tissue impedance characterization during the cardiac cycle IEEE Trans. Biomed. Eng. 58 3376-85

[26] Adams K L, Engelbrektsson J, Voinova M, Zhang B, Eves D J, Karlsson R, Heien M L, Cans A-S and Ewing A G 2010 Steady-state electrochemical determination of lipidic nanotube diameter utilizing an artificial cell model Anal. Chem. 82 1020-6

[27] Vangheem E 2004 Instantaneous impedance measurements on aluminium using a Schroeder multisine excitation signal Electrochim. Acta 49 2919-25

[28] Sanchez B, Vandersteen G, Bragos R and Schoukens J 2012 Basics of broadband impedance spectroscopy measurements using periodic excitations Meas. Sci. Technol. at press

[29] Simon G and Schoukens J 2000 Robust broadband periodic excitation design IEEE Trans. Instrum. Meas. 49 270-4

[30] Goodwin G C, Welsh J, Feuer A and Derpich M 2006 Utilizing prior knowledge in robust optimal experiment design 14th IFAC Symp. on System Identification pp 1358-63 
[31] Rojas C R, Welsh J S, Goodwin G C and Feuer A 2007 Robust optimal experiment design for system identification Automatica 43 993-1008

[32] Rojas C R, Agüero J C, Welsh J S and Goodwin G C 2008 On the equivalence of least costly and traditional experiment design for control Automatica 44 2706-15

[33] Rojas C R, Aguero J and Welsh J 2012 Robustness in experiment design IEEE Trans. Autom. Control 57 860-74

[34] Zarrop M and Goodwin G C 1975 Comments on optimal inputs for system identification IEEE Trans. Autom. Control 20 299-300

[35] Ng T, Payne R L and Goodwin G C 1977 On maximal accuracy estimation with output power constraints IEEE Trans. Autom. Control 133 133-4

[36] Ng T, Goodwin G C and Soderstrom T 1977 Optimal experiment design for linear systems with input output constraints Automatica 13 571-7

[37] Ljung L 1987 System Identification: Theory for the User (Englewood Cliffs, NJ: Prentice-Hall)

[38] Kanai H, Sakamoto K and Haeno M 1983 Electrical measurement of fluid distribution in human legs: estimation of extra- and intra-cellular fluid volume J. Microw. Power 18 233-43

[39] Randles J E B 1947 Kinetics of rapid electrode reactions Discuss. Faraday Soc. 111

[40] Sanchez B, Vandersteen G, Bragos R and Schoukens J 2011 Optimal multisine excitation design for broadband electrical impedance spectroscopy Meas. Sci. Technol. 22115601

[41] Åström K and Eykhoff P 1971 System identification-a survey Automatica 7 123-62

[42] Goodwin G C and Payne R L 1977 Dynamic System Identification: Experiment Design and Data Analysis vol 136 (New York: Academic)

[43] Curto R and Fialkow L 1991 Recursiveness, positivity and truncated moment problems Houst. J. Math. 17 603-35

[44] Grant M and Boyd S 2008 Graph implementations for nonsmooth convex programs Recent Advances in Learning and Control ed V Blondel et al (Berlin: Springer) pp 95-110

[45] Grant M and Boyd S 2011 CVX: Matlab software for disciplined convex programming, version 1.21

[46] Guillaume P, Schoukens J, Pintelon R and Kollar I 1991 Crest-factor minimization using nonlinear Chebyshev approximation methods IEEE Trans. Instrum. Meas. 40 982-9

[47] Gaw R, Box R and Cornish B 2011 Bioimpedance in the assessment of unilateral lymphedema of a limb: the optimal frequency Lymphatic Res. Biol. 9 93-9

[48] Sarro E, Lecina M, Fontova A, Sola C, Godia F, Cairo J J and Bragos R 2012 Electrical impedance spectroscopy measurements using a four-electrode configuration improve on-line monitoring of cell concentration in adherent animal cell cultures Biosens. Bioelectron. 31 257-63

[49] Reitinger S, Wissenwasser J, Kapferer W, Heer R and Lepperdinger G 2012 Electric impedance sensing in cell-substrates for rapid and selective multipotential differentiation capacity monitoring of human mesenchymal stem cells Biosens. Bioelectron. 34 63-9

[50] Cole K S 1940 Permeability and impermeability of cell membranes for ions Cold Spring Harb. Symp. Quant. Biol. 8 110-22

[51] Kun S and Peura R A 1999 Selection of measurement frequencies for optimal extraction of tissue impedance model parameters Med. Biol. Eng. Comput. 37 699-703

[52] Ward L and Cornish B H 2004 Multiple frequency bioelectrical impedance analysis how many frequencies to use? Proc. Int. Conf. Electrical Bioimpedance Electrical Impedance Tomography (Gdansk) (Gdansk University of Technology) pp 321-4

[53] Sanchez B, Bragos R and Vandersteen G 2011 Influence of the multisine excitation amplitude design for biomedical applications using impedance spectroscopy 33rd Annu. Int. Conf. of the IEEE Engineering in Medicine and Biology Society pp 3975-8

[54] Godfrey K R, Barker H A and Tucker A J 1999 Comparison of perturbation signals for linear system identification in the frequency domain IEEE Proc., Control Theory Appl. $146535-48$

[55] Schoukens J and Dobrowiecki T 1998 Design of broadband excitation signals with a user imposed power spectrum and amplitude distribution Proc. IEEE Instrumentation and Measurement Technology Conf. vol 2, pp 1002-5

[56] Seo A, Rys M and Konz S 2001 Measuring lower leg swelling: optimum frequency for impedance method Med. Biol. Eng. Comput. 39 185-9

[57] Osypka M and Gersing E 1995 Tissue impedance spectra and the appropriate frequencies for EIT Physiol. Meas. 16 (Suppl A) A49-55

[58] Schoukens J, Pintelon R and Rolain Y 2000 Broadband versus stepped sine FRF measurements IEEE Trans. Instrum. Meas. 49 275-8

[59] Spiridonakos M and Fassois S 2009 Parametric identification of a time-varying structure based on vector vibration response measurements Mech. Syst. Signal Process. 23 2029-48

[60] Schoukens J, Rolain Y and Pintelon R 2006 Leakage reduction in frequency-response function measurements IEEE Trans. Instrum. Meas. 55 2286-91

[61] Pintelon R, Schoukens J, Vandersteen G and Barbé K 2010 Estimation of nonparametric noise and FRF models for multivariable systems: part I. Theory Mech. Syst. Signal Process. 24 573-95

[62] Pintelon R, Schoukens J, Vandersteen G and Barbé K 2010 Estimation of nonparametric noise and FRF models for multivariable systems: part II. Extensions, applications Mech. Syst. Signal Process. 24 596-616

[63] Lataire J, Pintelon R and Louarroudi E 2012 Non-parametric estimate of the system function of a time-varying system Automatica 48 666-72 